\title{
ACE 29
}

\section{LA VEGETACIÓN EN EL CONFORT MICRO CLIMÁTICO. COMPARACIÓN DE ESPECIES DEL CLIMA MEDITERRÁNEO DE BARCELONA, ESPAÑA}

Gilkauris Maria Rojas Cortorreal, Jaume Roset y Francesc Navés

Cómo citar este artículo: ROJAS, G.M.; ROSET, J. y NAVÉS, F. La vegetación en el confort micro climático. Comparación de especies del clima mediterráneo de Barcelona, España [en línea] Fecha de consulta: dd-mm-aa. En: ACE: Architecture, City and Environment = Arquitectura, Ciudad y Entorno, 10 (29): 59-84, 2015. DOI: 10.5821/ace.11.29.3589. ISSN: 1886-4805.

\section{ACE:




\title{
ACE 29
}

Electronic offprint

Separata electrónica

THE VEGETATION IN THE MICROCLIMATE COMFORT. COMPARISON OF SPECIES OF MEDITERRANEAN CLIMATE OF BARCELONA, SPAIN

Key words: Mediterranean climate; urban vegetation; urban comfort, vegetation species.

\begin{abstract}
It is well known that vegetation is an element that allows to reduce heat input to the microclimate, previous studies have explored how urban vegetation improve certain aspects. But they have not made many studies of the variability of the species based on comfort and microclimate. Each species has different characteristics, so their behavior and contributions to the microclimate are different, hence arises the need to know the variables, indicators and contributions of each species. The objective of this study is to analyze the quality of urban micro climate due to decreasing the amount of heat input by vegetation. To know in detail the characteristics of each tree species and make some technical specifications for use of urban design. With possible interpolation with other climates due to the variability of species in the city of Barcelona.
\end{abstract}

The methodology used was qualitative and quantitative. urban morphology and tree species were selected, the selection criteria used were the variability of species, the short distance between them allowing more versatility when in situ measurements, the high demand of users hosted this urban structure, guidance and variability of pavements with the same type of species. After selecting a study of these tree species thus creating form data sheets was performed. an analysis of observation of the busiest urban structures of the area was performed. Measurements comfort parameters were performed. Qualitative results were: Woodland features, bioclimatic diversity of species, different kinds of shadows formed by branches and tree species. And quantitative were: internal / external differences in the tree canopy radiant

\section{ACE




\section{ACE 29}

\section{Electronic offprint}

\section{Separata electrónica}

temperature (from now on $\mathrm{Tr}$ ), luminance (from now on L ) and illuminance (from now on $\mathrm{E}$ ), little change in the air temperature (from now on $\mathrm{Ta}$ ) and significant variations in relative humidity (from now on HR ) during the day. Resulting qualitative values of the benefits of tree species, quality improvement in urban structure because of the amount of difference of thermal input and the recommendation of the use of urban concrete pavement with grass. The conclusions of how vegetation were able to create urban comfort and at what levels. 


\title{
LA VEGETACION EN EL CONFORT MICRO CLIMÁTICO. COMPARACIÓN DE ESPECIES DEL CLIMA MEDITERRANEO DE BARCELONA, ESPAÑA
}

\author{
ROJAS CORTORREAL, Gilkauris Maria ${ }^{1}$ \\ ROSET, Jaume \\ NAVÉS, Francesc
}

Remisión inicial: 23-05-2014

Remisión final: 28-09-2015

Palabras clave: Clima mediterráneo; vegetación urbana; confort urbano, especies vegetación.

Key words: Mediterranean climate; urban vegetation; urban comfort, vegetation species.

\section{Resumen estructurado}

Es bien conocido que la vegetación es un elemento que logra disminuir aportaciones térmicas al microclima, en estudios previos se ha analizado como la vegetación mejora ciertos aspectos urbanos. Pero no se han realizados muchos estudios de la variabilidad de las especies en base al confort y el micro clima. Cada especie posee características diferentes, por lo que su comportamiento y aportaciones al microclima son distintas, de aquí nace la necesidad de conocer las variables, indicadores y aportaciones de cada especies. El objetivo de este estudio es el análisis de la calidad del micro clima urbano debido a disminución de la cantidad de aportaciones térmicas por la vegetación. Conocer en detalle las características de cada especie arbórea y realizar unas fichas técnicas para uso de diseño urbano. Con posible interpolación con otros climas debido a la variabilidad de especies en la ciudad de Barcelona.

La metodología que se utilizó fue la cualitativa y cuantitativa. Se seleccionaron especies arbóreas y morfología urbana, el criterio de selección utilizado fueron la variabilidad de especies, la poca distancia entre ellas permitiendo más versatilidad a la hora de mediciones "in situ", la gran demanda de usuarios que alberga esta estructura urbana, la orientación y la variabilidad de pavimentos con los mismos tipos de especies. Luego de la selección se realizó un estudio de estas especies arbóreas creando de esta forma fichas técnicas. Se realizó un análisis de observación de las estructuras urbanas más concurridas de la zona. Se realizaron mediciones de los parámetros de confort ${ }^{2}$. Los resultados obtenidos cualitativos fueron: características del arbolado, diversidad de especies bioclimáticas, diferentes tipos de sombras, ramaje y forma por especies arbóreas. Y cuantitativos fueron: diferencias interior / exterior de la

\footnotetext{
${ }^{1}$ Gilkauris Maria Rojas Cortorreal: Estudiante del Programa de Doctorado "Ámbitos de Investigación en la Energía y el Medio Ambiente en la Arquitectura" del Departamento de Construcciones Arquitectónicas I. Escuela Técnica Superior de Arquitectura de Barcelona, Universidad Politécnica de Cataluña. Email de contacto: gilkaurisrojas@gmail.com

${ }^{2}$ Los parámetros del confort son temperatura del aire [Ta], temperatura radiante [ $\left.\mathrm{Tr}\right]$, humedad relativa [Hr], radiación $[R]$, Luminancia [L] e lluminancia [E].
} 
copa del árbol de temperatura radiante (de ahora en adelante $T r)^{3}$, luminancia (de ahora en adelante $\mathrm{L})^{4}$ e iluminancias (de ahora en adelante $\left.\mathrm{E}\right)^{5}$, escasa variación en la temperatura del aire (de ahora en adelante $\mathrm{Ta})^{6}$ y variaciones significativa de la humedad relativa (de ahora en adelante $\mathrm{Hr})^{7}$ en el transcurso del día. Dando como resultado valores cualitativos de los beneficios que aportan las especies arbóreas, mejora de calidad en la estructura urbana debido a la cantidad de diferencia de aportaciones térmicas y la recomendación del uso del pavimento urbano el hormigón con césped. Se presentan conclusiones de cómo la vegetación logra crear confort urbano y a qué niveles.

El objetivo del presente artículo es estudiar de forma cualitativa y cuantitativa la vegetación, como esta influye en el confort y el micro clima a través de la disminución de aportaciones térmicas. Conocer en detalle las características de cada especie arbórea para realizar unas fichas técnicas de diseño urbano. De forma que se pueda hacer una posible interpolación con otros climas. Siendo el estudio factible debido a la variabilidad de especies arbóreas que existe en Barcelona. Por otra parte, la información obtenida de la vegetación en clima mediterráneo nos permitirá intentar prever los cambios confort micro climático que podría obtenerse en cada especie arbórea.

\section{Estado del arte}

Las investigaciones realizadas sobre el micro clima urbano, confort y vegetación se han desarrollado en diferentes tipos de ciudades (climas distintos). Existen un gran número de investigaciones a nivel de tesis (máster o doctoral) y artículos, los cuales tienen en común el confort urbano.

Cabe destacar entre las publicaciones analizadas: tres (3) tesis doctorales desarrolladas en Barcelona. La primera que podemos citar se realizó en el año 1999, por José Manuel Ochoa de la Torre con el titulo "La Vegetación como Instrumento para el Control Micro climático"(Ochoa de la Torre 1999), en el 2007 Cynthia Echave Martínez realizó la tesis "Ver des de la ciudad. Potencial de habitabilidad térmica en espacios urbanos"(Echave Martinez 2007) y en el 2010 Mireya Alicia Rosas Lusett presentó su investigación "El confort ambiental de las circulaciones peatonales en el litoral costero de Barcelona, España"(Rosas Lusett 2010). Por otra parte en la ciudad de Madrid se realizó en el 2012 la tesis doctoral "El microclima urbano en los espacios abiertos. Estudio de casos en Madrid" por Inina Tumini.(Tumini 2012).

Los artículos de investigaciones realizadas desde 1988 a 2013 cabe mencionar los siguientes: "Microclimatic conditions of urban public spaces in a tropical city "(Melo Barbirato, Carnaúba Torres and De Almeida Lisboa 1988), "Evaluación energética - Ambiental del Bosque Urbano: Desarrollo y puesta a punto de un método de análisis“"(Cantón, Cortegoso and De Rosa 2000), "City of Honolulu, Hawai'i, Municipal Forest Resource Analysis"(Vargas et al. 2007), "The effect of tree shade and grass on surface and globe temperatures in an urban area"(Armson, Stringer and Ennos 2012), "Thermal comfort conditions of shaded outdoor spaces in hot and humid

\footnotetext{
${ }^{3}$ Temperatura radiante ( $\left.\mathrm{Tr}\right)$ : es la temperatura que emite un cuerpo. Unidad ${ }^{\circ} \mathrm{C}$.

${ }^{4}$ Luminancia (L): intensidad radiante emitida por unidad de una superficie aparente determinada. Unidad $\mathrm{cd} / \mathrm{m}^{2}$.

${ }^{5}$ Iluminancia (E): flujo radiante que llega a una superficie determinada. Unidad lux, $1 \mathrm{x}=\mathrm{Im} / \mathrm{m}^{2}$.

${ }^{6}$ Temperatura del aire (Ta): temperatura o magnitud de las moléculas del aire. Unidad ${ }^{\circ} \mathrm{C}$

${ }^{7}$ Humedad relativa $(\mathrm{Hr})$ : cantidad de vapor de agua en el aire. Unidad \%
} 
climate of Malaysia"(Makaremi et al. 2012), "Bioclimate provide by shade trees as factor in urban and architectural planning in tropical climates - The case of Campinas, Brazil."(Abreu, Labaki and Matzarakis 2012) y "Permeabilidad del arbolado urbano a la radiación solar: estudio de dos especies representativas en entornos urbanos de baja densidad del área metropolitana de Mendoza, Argentina"(Arboit 2013). En estas publicaciones se habla del arbolado; sus características, sistemas de plantación, pero no de los beneficios de confort que puede ofrecer.

\section{Metodología}

La metodología a utilizar será cuantitativa y cualitativa. Los procedimientos a realizar en la investigación serán los siguientes:

- Selección de especies arbóreas. Los criterios de selección fueron las principales especies de mayor demanda en uso urbano en Barcelona, tomando como base el libro "Gestión del arbolado viario de Barcelona"(De Barcelona 2011). Se estudiaron y desglosaron las características (origen, zona bioclimática, forma, ramaje, sombra, entre otros) de estas especies.

- Selección de morfología urbana. Los criterios de selección fueron la distancia mínima entre especies seleccionadas, permitiendo más versatilidad a la hora de mediciones "in situ", la gran demanda de usuarios que alberga este morfologia urbano (Passeig de Sant Joan), la orientación y la variabilidad de tipos de pavimentos con los mismos tipos de especies.

- Selección de tipo de pavimentos a evaluar, donde el criterio fue la elección de los más usados en zona de recreación de Barcelona.

- Elección de los puntos de mediciones, tomando como criterio la especie de mayor uso o demanda por el usuario y el tipo de pavimento.

- Se realizaron mediciones "in situ" en cada punto seleccionado (dentro y fuera de la sombra del arbolado) de los parámetros de confort en la ciudad de Barcelona.

- Se analizan los resultados obtenidos de cada punto, pavimento y especie arbórea.

\section{$\underline{\text { Conclusiones }}$}

Entre los resultados obtenidos podemos resaltar dos conclusiones principales: Que la vegetación en la morfología urbana logra variaciones en la temperatura radiante (Tr) de hasta $25^{\circ} \mathrm{C}$ de diferencia. En el estudio de los tipos de pavimentos el más recomendado para el uso en el diseño urbano es el hormigón con césped debido a la disminución de las aportaciones térmica.

\section{Originalidad}

La originalidad del estudio es que se analizó la herramienta de protección solar número uno que es la vegetación. Donde el objetivo principal es conocer cuales especies arbóreas ofrece mayor calidad a la morfología urbana y cuanta disminución de aportaciones térmicas crea al usuario. 


\section{Introducción}

La vegetación forma parte del ecosistema desde la creación del mundo. De igual forma podemos ver como el ser humano a través de la historia, toma la vegetación como inspiración para soluciones en general. En la actualidad la morfología urbana se está viendo afectado por los problemas de la isla de calor. Se han realizado estudios para lograr conocer los factores que lo afectan y de esta forma mejorar estos espacios a nivel de confort y vegetación.

De los trabajos realizados cabe destacar tres (3) tesis doctorales realizadas para la ciudad de Barcelona: En el 1999 Ochoa de la Torre(Ochoa de la Torre 1999) realizó una tesis doctoral que estudiaba los elementos que afectan estos espacios, realizando mediciones y cálculos para de esta forma conocer el balance de confort. En el 2007 Echave Martínez(Echave Martinez 2007) estudió la morfología urbana como modelo de habitabilidad. En el 2010 Rosas Lusett(Rosas Lusett 2010) tuvo un enfoque más en el ámbito costero y el confort que se busca en el mismo.

Los artículos de investigaciones realizadas desde 1988 al 2013 cabe mencionar los siguientes: "Microclimatic conditions of urban public spaces in a tropical city"(Barbirato et al, 1988), "Evaluación energética - Ambiental del Bosque Urbano: Desarrollo y puesta a punto de un método de análisis" (Cantón et al, 2000), "City of Honolulu, Hawai'i, Municipal Forest Resource Analysis"(Vargas et al. 2007), "The effect of tree shade and grass on surface and globe temperatures in an urban area"(Armson et al, 2012), "Thermal comfort conditions of shaded outdoor spaces in hot and humid climate of Malaysia" (Makaremi et al, 2012), "Bioclimate provide by shade trees as factor in urban and architectural planning in tropical climates - The case of Campinas, Brazil" (Abreu et al, 2012) y "Permeabilidad del arbolado urbano a la radiación solar: estudio de dos especies representativas en entornos urbanos de baja densidad del área metropolitana de Mendoza, Argentina" (Arboit, 2013) .

Como podemos ver a través de los años la vegetación, que es un elemento natural pasa a ser la herramienta número uno para solucionar o mitigar estas problemáticas. En este punto entra la necesidad del análisis ${ }^{8}$ de la vegetación y que niveles de protección que ofrece a través de la disminución de las aportaciones térmicas de un espacio determinado. Para poder darle soporte a esta hipótesis, se realizaron unas mediciones in situ de los árboles más utilizados en el diseño urbano.

\section{Elementos a evaluar}

\subsection{Climáticos}

El factor principal que afecta directamente el confort es el clima. Por lo que para realizar este análisis, debemos conocer las características ${ }^{9}$ de la ciudad de Barcelona (Tabla 1).

\footnotetext{
${ }^{8}$ Dentro de las investigaciones realizadas en Barcelona, cabe mencionar que se sigue la línea de investigación que realizó Ochoa de la Torre, que son mediciones en el ámbito urbano de la vegetación.

${ }^{9}$ Características de ubicación, localización, el clima, la radiación, la vegetación y la zona urbana.
} 
Tabla 1. Características de la ciudad de Barcelona

\begin{tabular}{|c|c|c|c|c|}
\hline \multicolumn{5}{|c|}{ Ciudad de Barcelona } \\
\hline \multicolumn{5}{|c|}{ Cataluña, España } \\
Latitud de 41.5 N 2.1 E \\
Clima \\
\hline Tipo & $\begin{array}{c}\text { Temperatura } \\
\text { medía máxima }\end{array}$ & $\begin{array}{c}\text { Temperatura medía } \\
\text { mínima }\end{array}$ & $\begin{array}{c}\text { Precipitación } \\
\text { Media }\end{array}$ & Humedad \\
\hline clima mediterráneo & $20.0^{\circ} \mathrm{C}$ & $16.5^{\circ} \mathrm{C}$ & $599 \mathrm{~mm}$ & $64 \%$ \\
\hline
\end{tabular}

Fuente: Elaboración propia. Datos suministrados por Atlas de radiació solar catalunya(Catalunya 2001)

El clima mediterráneo podemos definirlo con un clima seco, cálido en verano y húmedo en invierno. Con una oscilación moderada (Navés, 2005). Para conocer un poco más sobre este clima realizamos el diagrama de Gaussen ${ }^{10}$, se tomaron los datos de la media registradas de 20 a 30 años. El resultado obtenido del diagrama fue que la ciudad de Barcelona posee primavera e inviernos con mayores lluvias siendo esta estación húmeda y verano de lluvias muy escasas dando como resultado estaciones muy secas. Luego de este análisis podemos concluir que la ciudad posee altas temperaturas en el mes de Julio, siendo una de las estaciones más seca. Tomando como factor principal la temperatura, decidimos realizar las mediciones en el mes de Julio, ya que es el mes que podría aportarnos mayor información de la protección que genera la vegetación en el ámbito urbano.

\subsection{Vegetación}

La vegetación es un elemento que aporta ciertas características dependiendo de su zona climática de su procedencia. Las características climáticas infieren en su forma de adaptación. Para la selección de las especies de la ciudad de Barcelona, se tomo como base "La gestión del arbolado viario de Barcelona" (De Barcelona, 2011) donde se desglosan todas las especies de uso urbano de la ciudad. Se seleccionaron para el análisis las más predominantes en el diseño urbano (Tabla 2). Las principales características a considerar son: la forma, el ramaje y la sombra. Ya que a través de ellas es que podemos lograr la protección solar.

\footnotetext{
${ }^{10}$ Diagrama de Gaussen: este diagrama permite identificar los períodos del año más secos y húmedos.
} 
$\begin{array}{ll}\text { Architecture, City, and Environment } & (5) \\ \text { Arquitectura, Ciudad y Entorno }\end{array}$

Tabla 2. Especies seleccionadas para el estudio en la ciudad de Barcelona

\begin{tabular}{|c|c|c|c|c|c|}
\hline \multicolumn{6}{|c|}{ Ciudad de Barcelona } \\
\hline \multicolumn{2}{|c|}{ Especies / Nombre } & Zona bioclimática & Forma & Ramaje & Sombra \\
\hline Científico & Común & & & & \\
\hline Celtis australis & Almez & Bosque esclerófilos & Esférica & Expandido & Densa \\
\hline Tipuana tipu & Palo Rosa & $\begin{array}{c}\text { Bosques } \\
\text { semicaducifolios }\end{array}$ & Esférica & Expandido & $\begin{array}{l}\text { Media/ } \\
\text { medio }\end{array}$ \\
\hline Celtis siliquastrum & Árbol del amor & Bosque esclerófilos & $\begin{array}{l}\text { Esférica/ } \\
\text { irregular }\end{array}$ & Expandida & $\begin{array}{l}\text { Media/ } \\
\text { medio }\end{array}$ \\
\hline $\begin{array}{c}\text { Koelreuteria } \\
\text { paniculata }\end{array}$ & $\begin{array}{l}\text { Árbol de los } \\
\text { faroles }\end{array}$ & $\begin{array}{l}\text { Bosques húmedos } \\
\text { templados }\end{array}$ & $\begin{array}{l}\text { Esférica/ } \\
\text { irregular }\end{array}$ & Tortuoso & $\begin{array}{l}\text { Media/ } \\
\text { medio }\end{array}$ \\
\hline $\begin{array}{l}\text { Platanus } x \\
\text { hispanica }\end{array}$ & $\begin{array}{l}\text { Platano de } \\
\text { sombra }\end{array}$ & - & Ovoidal & Expandida & $\begin{array}{l}\text { Densa/ } \\
\text { grande }\end{array}$ \\
\hline Tilia platyphyllos & $\begin{array}{l}\text { Tilo de hojas } \\
\text { grandes }\end{array}$ & Bosque verdes & Ovoidal & Expandida & $\begin{array}{l}\text { Densa/ } \\
\text { grande }\end{array}$ \\
\hline $\begin{array}{c}\text { Phoenix } \\
\text { canariensis }\end{array}$ & Palmera canaria & $\begin{array}{c}\text { Bosques } \\
\text { semicaducifolios }\end{array}$ & $\begin{array}{c}\text { Abanico/par } \\
\text { asol }\end{array}$ & Palmiforme & $\begin{array}{l}\text { Media/ } \\
\text { grande }\end{array}$ \\
\hline Pittosporum tobira & Pitospor & $\begin{array}{l}\text { Bosques húmedos } \\
\text { templados }\end{array}$ & Esférica & Expandida & $\begin{array}{l}\text { Densa/ } \\
\text { grande }\end{array}$ \\
\hline $\begin{array}{c}\text { Abelia } x \\
\text { grandiflora }\end{array}$ & Abelia & - & Ovoidal & Expandida & $\begin{array}{l}\text { Medio/ } \\
\text { medio }\end{array}$ \\
\hline
\end{tabular}

Fuente: Elaboración propia. Datos suministrados por el libro Arquitectura del paisaje natural, 2005.(Navés 2005).

\subsection{Pavimentación}

Para este estudio se consideró importante evaluar los pavimentos más utilizados en la estructura urbana de Barcelona. Se eligieron tres tipos de pavimentos los cuales son el hormigón, hormigón/césped y cauce. Para la selección se consideró cuales eran más usados en zonas de recreación urbana como parques, áreas de bares y zonas de tránsito peatonal. Se eligieron especies arbóreas con distintos tipos de pavimentos, para evaluar las aportaciones térmicas al ambiente.

\section{Elección de punto de medición}

La zona de mediciones seleccionada fue Passeig Sant Joan y la calle Bailen (Figura 1). Los criterios de selección de ambas vías fueron: la variabilidad de pavimentos, de vegetación y por ser un lugar concurrente por un amplio público. En todo el transcurso de las dos (2) calles se seleccionaron cinco (5) punto de mediciones, haciendo un total de doce (12) mediciones. Que constan de un (1) puntos de medición en calle Bailen y cuatro (4) puntos de mediciones en Passeig Sant Joan (Tabla 3). 


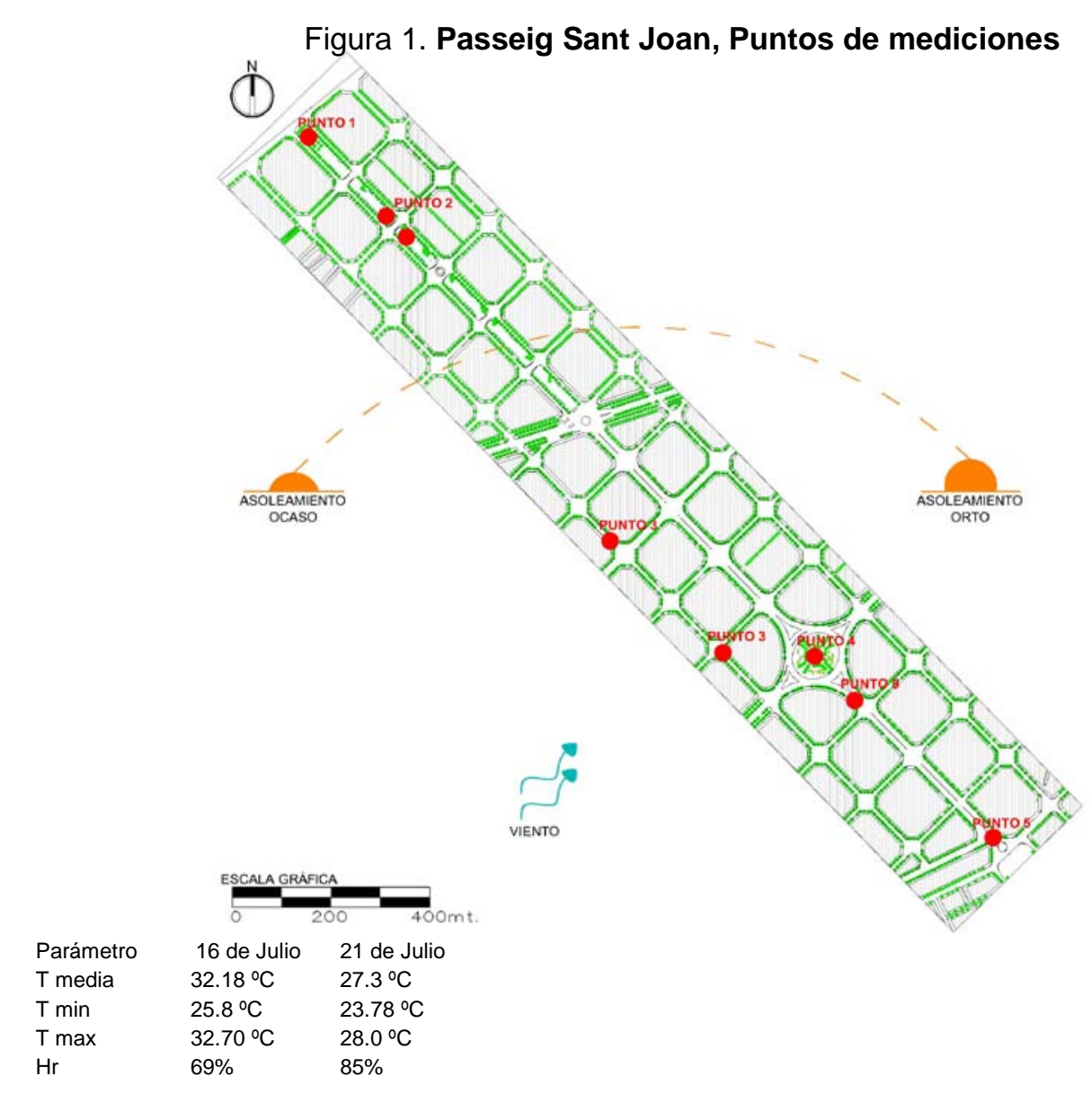

Fuente: Elaboración propia con base en Ajuntament de Barcelona.

Tabla 3. Características de los puntos de mediciones

\begin{tabular}{|c|c|c|c|c|c|}
\hline \multicolumn{2}{|c|}{ Punto } & Calle & Especie arbórea & Pavimento & Ubicación \\
\hline \multirow{2}{*}{\multicolumn{2}{|c|}{1}} & \multirow{4}{*}{$\begin{array}{l}\text { Passeig Sant } \\
\text { Joan }\end{array}$} & Alméz & \multirow{7}{*}{ Pavimento } & \multirow{4}{*}{$\begin{array}{c}\text { Desde Travessera de Gràcia } \\
\text { hasta Av. Diagonal }\end{array}$} \\
\hline & & & Tipuana & & \\
\hline \multirow{2}{*}{2} & 2.1 & & Árbol de los faroles & & \\
\hline & 2.2 & & Árbol del amor & & \\
\hline \multirow{3}{*}{3} & \multirow{3}{*}{3.1} & \multirow{3}{*}{ Bailen } & Alméz & & \multirow{3}{*}{$\begin{array}{c}\text { Desde Calle Valencia hasta } \\
\text { Gran Vía de Les Corts } \\
\text { Catalanes }\end{array}$} \\
\hline & & & Plátano de sombra & & \\
\hline & & & Tilos de hojas grandes & & \\
\hline & \multirow{3}{*}{4} & \multirow{5}{*}{$\begin{array}{l}\text { Passeig Sant } \\
\text { Joan }\end{array}$} & Alméz & \multirow{3}{*}{ Arena } & \multirow{5}{*}{$\begin{array}{l}\text { Desde Gran Vía de Les Corts } \\
\text { Catalanes hasta Ronda San } \\
\text { Pere (Arco del Triunfo) }\end{array}$} \\
\hline & & & Tipuana & & \\
\hline & & & Palmera Canaria & & \\
\hline \multirow[b]{2}{*}{5} & 5.1 & & Plátano de sombra & \multirow{2}{*}{$\begin{array}{l}\text { Pavimento / } \\
\text { césped }\end{array}$} & \\
\hline & 5.2 & & $\begin{array}{c}\text { Plátano de sombra, A. faroles y } \\
\text { arbustivos bajos }\end{array}$ & & \\
\hline
\end{tabular}

Fuente: Elaboración propia. 


\subsection{Características climáticas}

Las características climáticas del área de estudio son las expuestas en la figura 1. Podemos observar vientos predominantes noreste. El 16 de Julio del 2013 se realizaron las mediciones registrando temperaturas media de $32.18{ }^{\circ} \mathrm{C}$ y una radiación incidente de $953 \mathrm{~W} / \mathrm{m}^{2}$. Para el 21 de Julio del 2013 se realizó un recorrido fotográfico para el estudio del HDR se registraron una temperatura media de $27.3^{\circ} \mathrm{C}$ y una radiación incidente de $935 \mathrm{~W} / \mathrm{m}^{2}$.(Catalunya 2013)

\subsection{Características urbanas}

Passeig Sant Joan está ubicado en el distrito de l'Eixample derecho delimitado por Arco del triunfo y Travessera de Gràcia (sureste y noroeste), por la calle Roger de Flor y calle Bailen (noreste y suroeste). Conectando el distrito de Ciutat Vella con el distrito de Gràcia. Es una de las vías estructurales de la ciudad siendo actualmente un modelo de morfología urbano. Remodelado recientemente entre Arc de Triomf y plaza Tetuán (2010 - 2011), por la Arq. Lola Domènech (Domenech, 2008). Sus características urbanas son paseo urbano central con una sección de 50m de ancho, posee en los laterales (noreste y suroeste) dos calles principales de una vía, aceras perimetrales, gran variedad de vegetación, edificaciones de 8 niveles de vivienda y uso comercial con una altura estimada total de $24 \mathrm{~m}$. En su trayecto tiene una gran variedad de espacios de recreación para todas las edades. Por otra parte, la calle Bailen está delimitada por Ronda Sant Pere y Travessera de Gràcia (Sureste y Noroeste), por Passeig Sant Joan y calle Girona (Noreste y Suroeste). Sus características son calle de una vía de transito con una sección de $20 \mathrm{~m}$ de ancho, posee a los laterales aceras perimetrales, variedad de vegetación y edificaciones de 8 niveles de vivienda y uso comercial con una altura estimada total de $24 \mathrm{~m}$.

\subsection{Estrategias de medición}

Los puntos de estudio se situaron en el transcurso de Passeig Sant Joan y en la calle Bailen (Figura 1), desde Travessera de Gràcia en dirección al Arco del Triunfo. Las calles no poseen pendientes apreciables. Las mediciones fueron in situ. Se realizaron el 16 de Julio del 2013 desde las 9:00 hrs hasta las 18:00 $\mathrm{hrs}^{11}$. El 21 de Julio del 2013 se tomaron tres (3) rondas de fotografías, en tres (3) horas (9:00h, 13:00h y 17:00h) diferentes en los puntos de mediciones, para realizar un estudio de $\mathrm{HDR}^{12}$. Los criterios de selección para los dos días de estudios fueron las altas temperaturas y la alta incidencia de radiación solar. Los parámetros que se midieron fueron los del confort ${ }^{13}$ y un análisis de las imágenes realizadas en HDR, donde se estudiaron la luminancia e iluminancia. Se debe tomar en cuenta que el estudio de Luminancia e lluminancia se realizó para de igual forma observar el paso de la radiación solar en la vegetación. Los instrumentos de mediciones fueron: Cámara Nikon P90, 2 Sondas de mediciones modelos Testo 175H1, Termo radiante HIBOK 19, 2 luxómetros modelo LT lutron LX-101 y Kaise MS6610 y un termo anemómetro AZ8903. Las mediciones se realizaron a 1

\footnotetext{
${ }^{11}$ Se consideró las horas de mayor incidencia de radiación solar y mayor uso del espacio.

12 HDR: High dynamic range (Imagen de alto rango dinámico).

${ }^{13}$ Los parámetros del confort son temperatura del aire [Ta], temperatura radiante [Tr], humedad relativa [Hr], radiación $[R]$, Luminancia [L] e lluminancia [E].
} 
metro del suelo.

\section{Desarrollo detallado de un caso significativo. Punto I}

El punto I de medición se encuentra delimitado por Travessera de Gracia y Calle de Sant Antoni María Claret. La morfología del espacio es un paseo central, forma rectangular, con una calle de una vía en cada lateral (Suroeste y Noreste), aceras perimetrales, variedad de vegetación y posee edificios de 8 niveles con una altura estimada de 24m. (Figura 2).

Figura 2. Passeig Sant Joan, Punto de medición 1, Almez y Tipuana

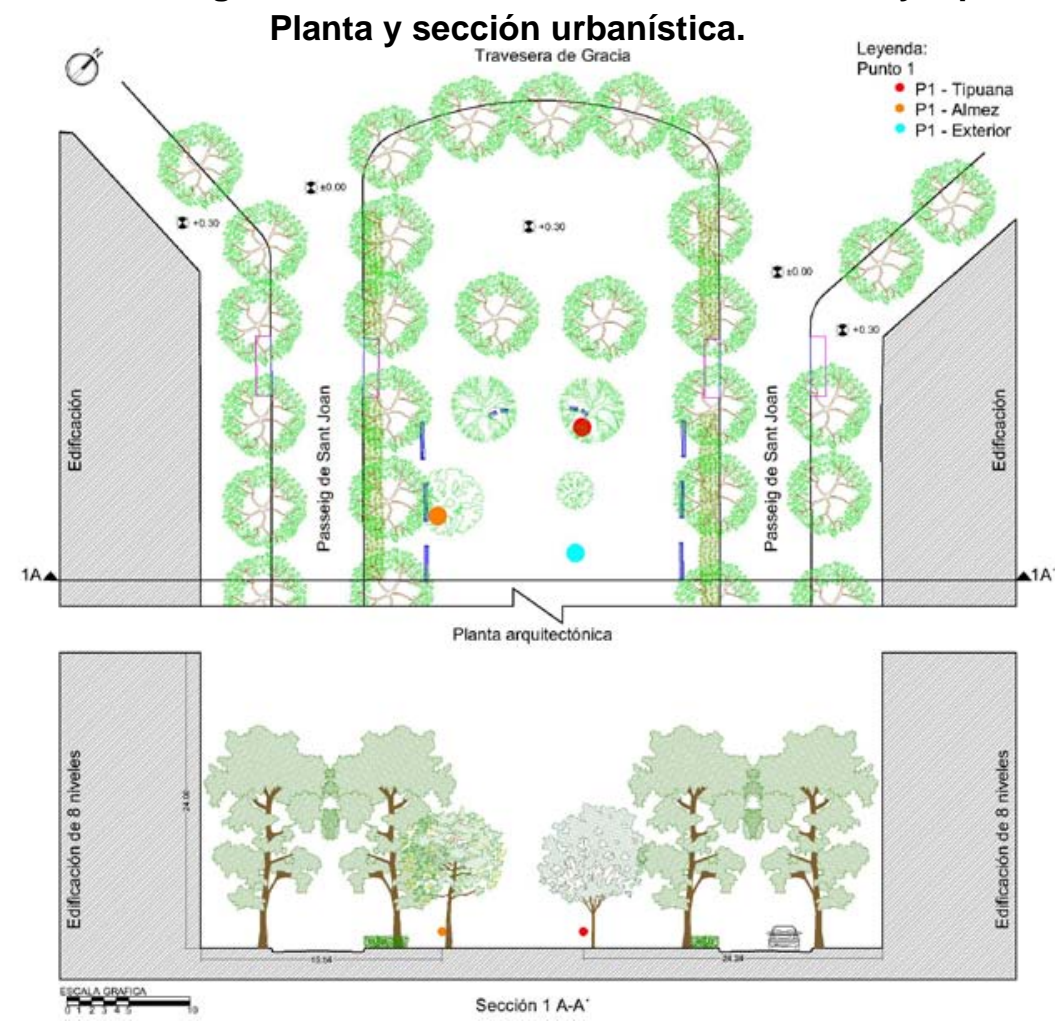

Fuente: Elaboración propia con base en Ajuntament de Barcelona.

Los criterios de selección de este punto fueron la abúndate demanda de uso por usuarios, la variedad de especies arbóreas y el tipo de pavimento que es el hormigón ${ }^{14}$. Las especies arbóreas de este punto de estudio fueron: Almez (Celtis australis) y Tipuana (Tipuana Tipu). Se realizaron unas fichas técnicas donde resume toda la información relevante de las dos especies (Figuras 3 y 5 ).

\footnotetext{
${ }^{14}$ Se tomó como referencia la investigación de Hypatia Caminero Peña(Caminero Peña 2012).
} 
Figura 3. Ficha técnica del Alméz. Análisis del arbolado

Análisis del Alméz.

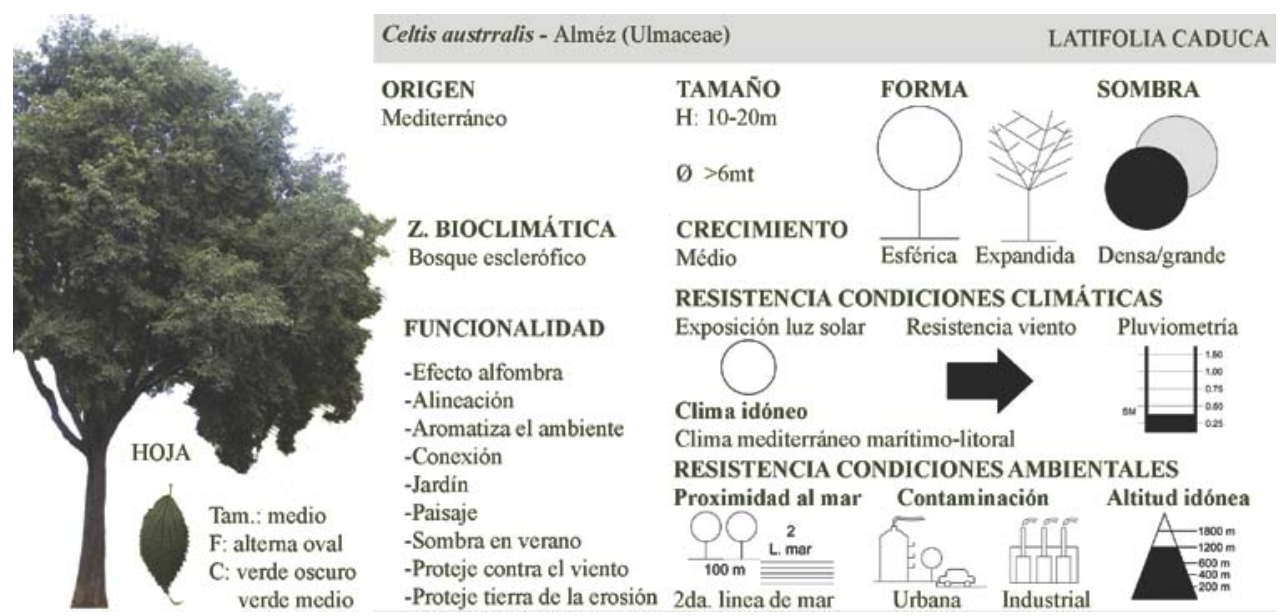

Fuente: Elaboración propia con base en libro el árbol en la jardinería y paisaje de Arq. Paisajista Francesc Navés.

Resultados de mediciones punto 1 - Almez. (Celtis australis): En la temperatura aire (Ta) se registraron valores no muy diferentes entre la Ta exterior ${ }^{15}$ y Ta interior ${ }^{16}$. (Gráfica 1 ) Estos registros fueron una Ta exterior máxima de $33.1^{\circ} \mathrm{C}$ y una Ta exterior mínima de $28.7^{\circ} \mathrm{C}$.

\section{Gráfica 1. Gráficas de temperatura y humedad en el interior y exterior del árbol. Temperatura del aire y Humedad relativa}

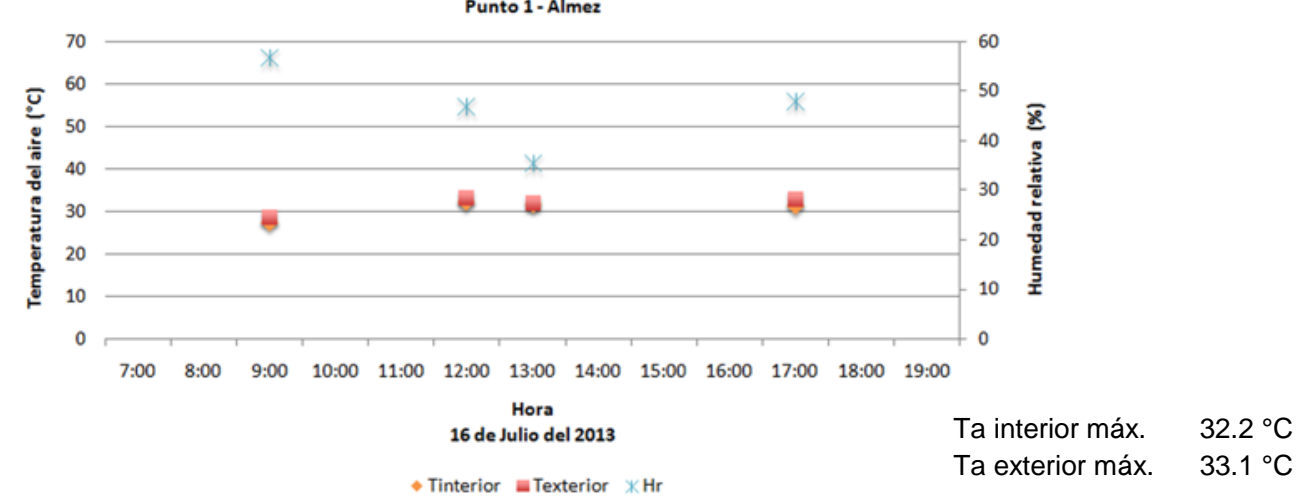

Fuente: Elaboración propia

Sin embargo la Ta interior máxima fue de $32.2^{\circ} \mathrm{C}$ y una Ta interior mínima de $27.5^{\circ} \mathrm{C}$. Dando una Ta exterior media de $31.7^{\circ} \mathrm{C}$ y una Ta interior media de $30.7^{\circ} \mathrm{C}$. Y una diferencia promedio de la Ta interior/exterior de casi $1{ }^{\circ} \mathrm{C}$. (Gráfica 1 ). La temperatura del aire registró muy pocas diferencias debido a la relación directa interior y exterior.

\footnotetext{
${ }^{15}$ Exterior: Se refiere a la medición realizada fuera de la copa de la sombra del árbol.

${ }^{16}$ Interior: Se refiere a la medición realizada bajo la copa de la sombra del árbol.
} 
En la humedad relativa se observó a primeras horas de la mañana una alta humedad, esto se debe a la acumulación de aportaciones de agua al ambiente durante la noche. Sin embargo, a medida que la Ta aumenta la humedad disminuye por la evaporación. Se registró una disminución de la Ta y un aumento de la humedad relativa a las 17:00h esto se debe a las aportaciones a la humedad absoluta por evapotranspiración de parte del árbol. (Gráfica 1).

El cuerpo humano regula su temperatura con la emisión de calor a través de la piel, cuando recibe aportaciones mayores a las que el mismo pueda soportar o manejar, entra en estado de estrés térmico. Por este motivo, cuantas menos aportaciones reciba en momento donde la radiación incidente es muy alta, mas en estado de confort podrá estar el cuerpo. Por esta razón la temperatura radiante es uno de los factores de mayor interés.

En las mediciones de la temperatura radiante ( $\mathrm{Tr}$ ) se puede percibir más los aportes que puede lograr la vegetación. Se registró una $\mathrm{Tr}$ exterior máxima de $59.4^{\circ} \mathrm{C}$ y una $\mathrm{Tr}$ exterior mínima de $27.9^{\circ} \mathrm{C}$. Y una $\mathrm{Tr}$ interior máxima fue de $35.8^{\circ} \mathrm{C}$ y una $\mathrm{Tr}$ interior mínima de $27.4^{\circ} \mathrm{C}$. La diferencia máxima registrada entre la $\mathrm{Tr}$ interior/exterior fue de $23.6^{\circ} \mathrm{C}$ a las $13: 00 \mathrm{hrs}$ (Gráfica 2). Con una $\mathrm{Tr}$ exterior media de $48.6^{\circ} \mathrm{C}$ y una $\mathrm{Tr}$ interior media de $33^{\circ} \mathrm{C}$. $\mathrm{Y}$ una diferencia promedio de la $\mathrm{Tr}$ interior/exterior de $15.6^{\circ} \mathrm{C}$.

\section{Gráfica 2. Gráfica de temperatura radiante en el interior y al exterior del árbol. Temperatura Radiante}

Punto 1- Almez

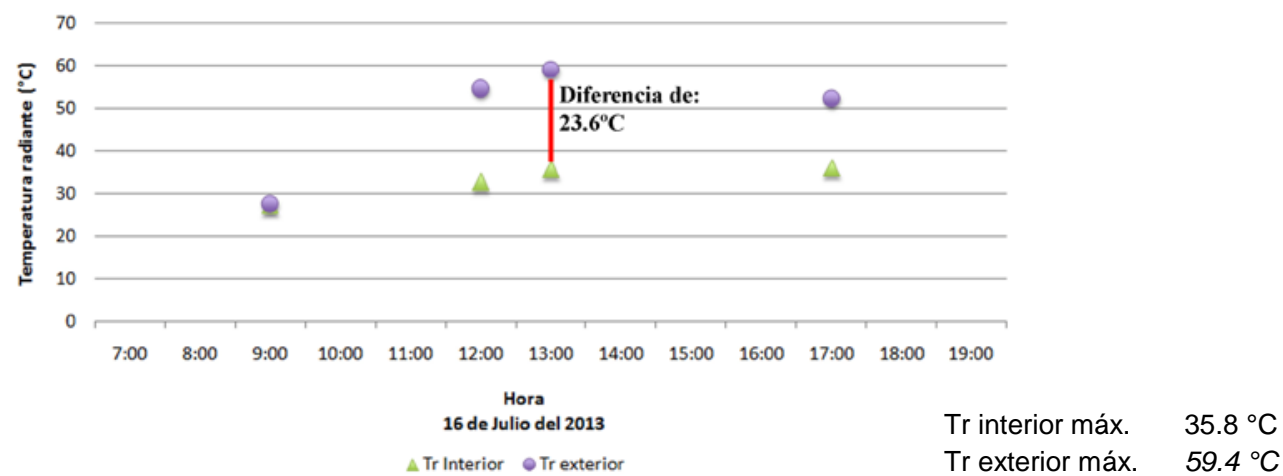

Fuente: Elaboración propia

Podemos observar como el calor emitido por radiación de la superficie, puede variar hasta en $23.6^{\circ} \mathrm{C}$ en relación a la superficie que está protegida por el árbol. Cuando en el ambiente existen aportaciones de temperatura de este tipo impide que el cuerpo disipe calor para poder regularse, tener una disminución de esta cantidad $\left(23.6^{\circ} \mathrm{C}\right)$, logra que bajo la sombra del árbol el usuario se encuentre en confort en relación al exterior.

En la iluminancia (E) Serra(Serra Florensa and Coch 1995) define los niveles de confort para una visualización de superficies con reflexión de $>0.2$ (muy iluminadas) de 20000 lux (>1000 $\mathrm{cd} / \mathrm{m}^{2}$ ). Siendo el valor de 1000 lux lo requerido para actividades con esfuerzo muy alto. Tomando como punto de partida los valores expresados por Serra analizamos los datos registrados. 
Se registró una $E$ exterior máxima de 107000 lx y una E exterior mínima de 5040 Ix. La E interior máxima registrada fue de $5830 \mathrm{~lx}$ y la E interior mínima fue de $1274 \mathrm{~lx}$, el usuario en el espacio se encuentra en confort. La $E$ interior/exterior se registró una diferencia de hasta $107300 \mathrm{Ix}$ a las 13:00 hrs. (gráfica 3). Con una E exterior media de 74060 Ix y E interior media de $2976 \mathrm{~lx}$. Llegando a tener una diferencia de la $\mathrm{E}$ interior/exterior media de $71084 \mathrm{~lx}$.

\section{Gráfica 3. Gráfica de iluminancia en el interior y al exterior del árbol lluminancia}

Punto 1-Almez

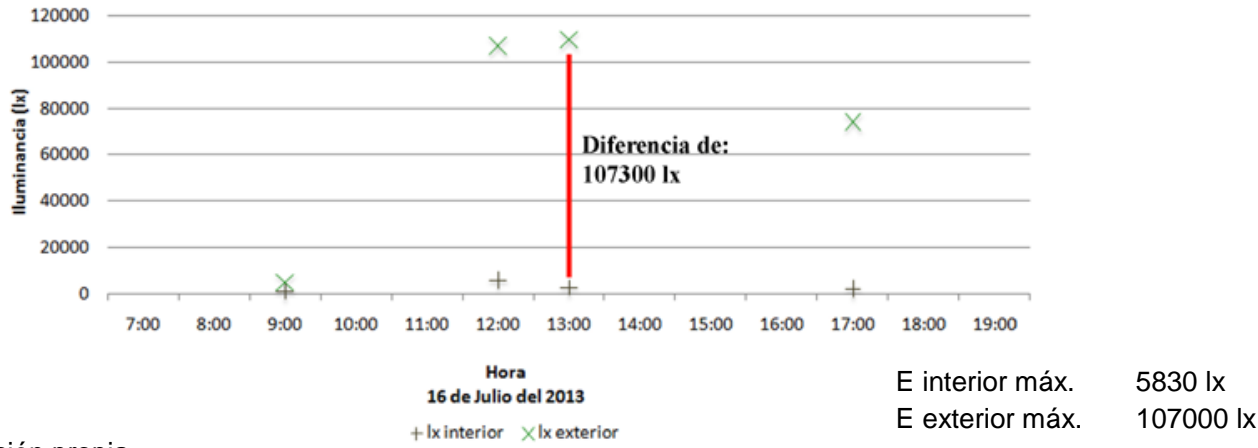

Fuente: Elaboración propia

La zona de confort para una actividad de mayor esfuerzo requiere una iluminancia de $1000 \mathrm{Ix}$, por lo que podemos ver que ese día el usuario estaba en disconfort visual por iluminancia ya que todos los datos exceden este valor. Sin embargo, bajo la sombra del árbol se logra disminuir esta $\mathrm{E}$, permitiendo que el ojo se adapte a una zona de confort.

Estudio de HDR(Jaloxa 2012): El análisis de la Luminancia nos permite ver el paso de luz debajo de la copa del árbol, como se comportaría la radiación solar y la disminución de los niveles de dis-confort. Serra(Serra Florensa and Coch 1995) define que para la visualización optima de trabajo normales se necesita $100-400 \mathrm{~cd} / \mathrm{m}^{2}$ (2000 lux).

Figura 4. Punto de medición 1. Luminancia. Almez. Hora 9:00am, 13:00 y 17:00 hr.

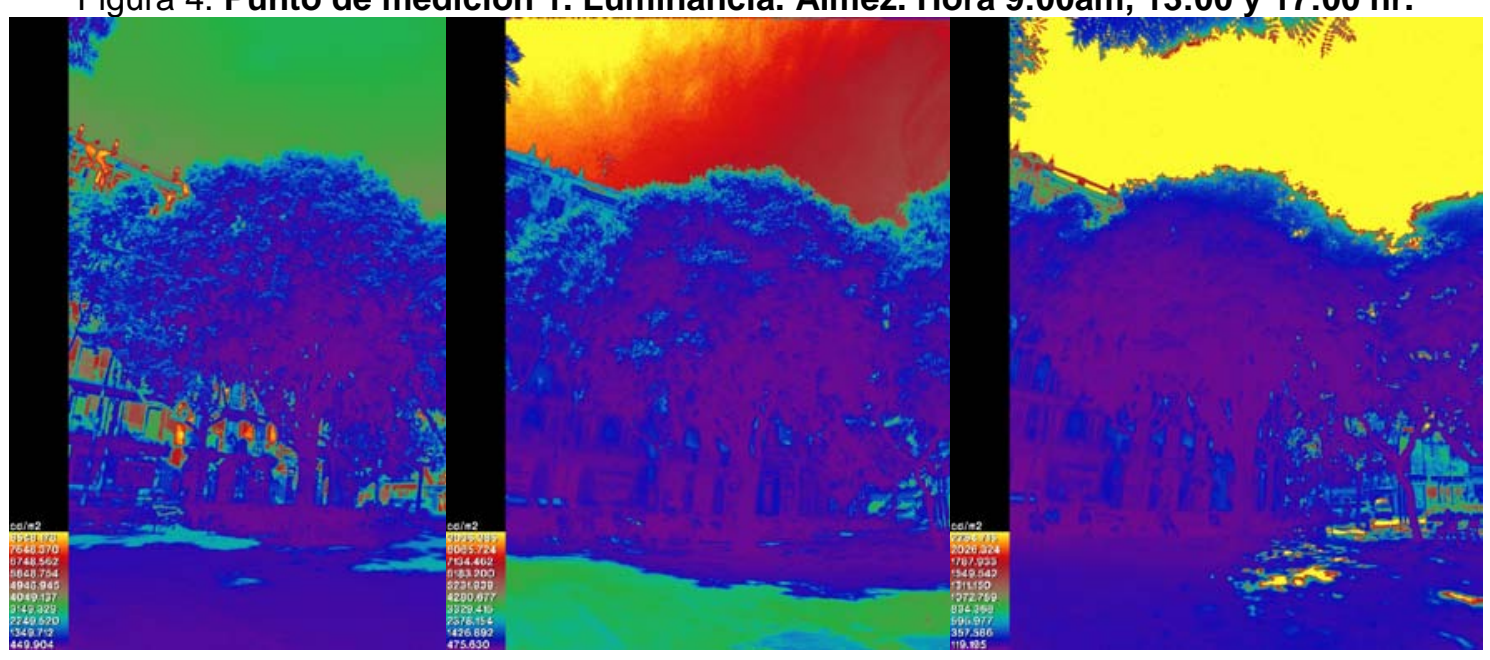

Fuente: Elaboración propia con base en Jaloxa. 
Este programa Jaloxa hace una relación de dos puntos en la imagen que capto el lente de la cámara, donde se registró mayor y menor luminancia. Tomando eso como base, el programa realiza una imagen en colores falsos. Esto hace que la interpretación de los diferentes tonos de luminancia sea más fácil. Por lo que la luminancia $(L)$ podría ser mayor, de lo que se registra en la foto. Se tomaron tres (3) imágenes a horas relativas a las mediciones.

Podemos observar en las imágenes que los valores de luminancia no exceden los rangos de confort, siempre estando en valores aceptables para la visión (Figura 4). Por otra parte, muestra como crea espacios de zonas donde se protege el pavimento, el tipo de sombra homogénea y la disposición de sus hojas evitando de esta forma el aporte de la $\mathrm{Tr}$ al ambiente.

Los arbustos protegen la zona inferior del árbol, en las horas donde el sol está en su orto y ocaso, logrando con esto la protección que la copa no puede dar en ese momento. Por otra parte, aporta datos de luminancia, es un medio a través del cual se ve como el ramaje y el tipo de hoja no permite el paso de la luz a ninguna hora del día. Por este motivo, los datos recolectados anteriormente demuestran una diferencia en la $\mathrm{Tr}$ interior con relación a la $\mathrm{Tr}$ exterior de hasta $23.6^{\circ} \mathrm{C}$.

Figura 5. Análisis del arbolado, ficha técnica Tipuana tipu - Palo rosa (Leguminosae Papilionaceae)

\section{LATIFOLIA CADUCA}

Análisis de la Tipuana.

\begin{tabular}{ll}
\hline ORIGEN & TAMAÑo \\
Brasil / Argentina & H: $10-15 \mathrm{~m}$
\end{tabular}

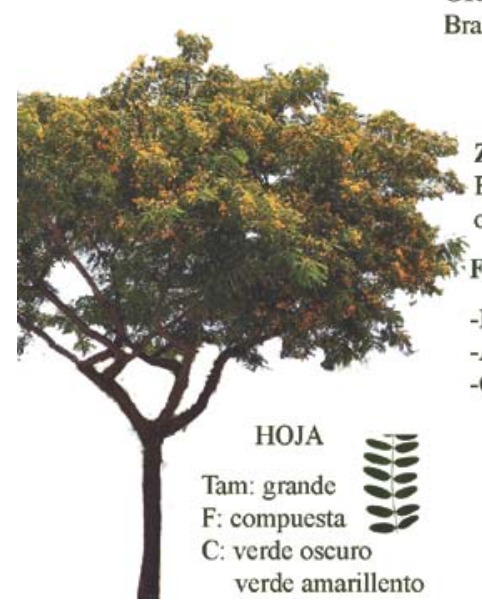

Bosques semicaducifolios

$\emptyset 4-6 \mathrm{mt}$

CRECIMIENTO

Rápido

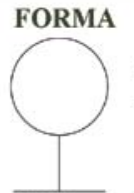

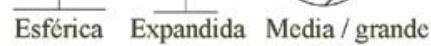

o caducifolios subtropicales RESISTENCIA CONDICIONES CLIMÁTICAS

FUNCIONALIDAD Exposición luz solar Resistencia viento Pluviometria

-Efecto alfombra

-Alineación

-Conf. 3 niveles de jardin

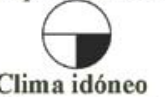

Clima subtropical mediterráneo

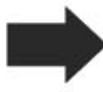

RESISTENCIA CONDICIONES AMBIENTALES

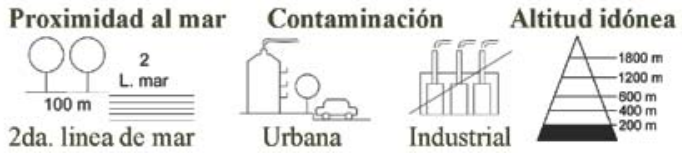

Fuente: Elaboración propia con base en libro el árbol en la jardinería y paisaje de Arq. Paisajista Francesc Naves.

Resultados mediciones punto 1 - Tipuana (Tipuana Tipu): En la temperatura aire se registró una Ta exterior máxima de $31.9{ }^{\circ} \mathrm{C}$ y una Ta exterior mínima de $28.68{ }^{\circ} \mathrm{C}$. La Ta interior máxima de $32.2^{\circ} \mathrm{C}$ y una Ta interior mínima de $27.5^{\circ} \mathrm{C}$. La Ta interior/exterior se mantiene constante, esto se debe a la relación directa con el espacio exterior. Dando una Ta exterior media de $31.7^{\circ} \mathrm{C}$ y una Ta interior media de $30.7^{\circ} \mathrm{C}$. Y una diferencia promedio de la $\mathrm{Ta}$ interior/exterior de casi $1{ }^{\circ} \mathrm{C}$. (Gráfica 4). La temperatura del aire registró muy pocas diferencias debido a la relación directa del interior y el exterior. 


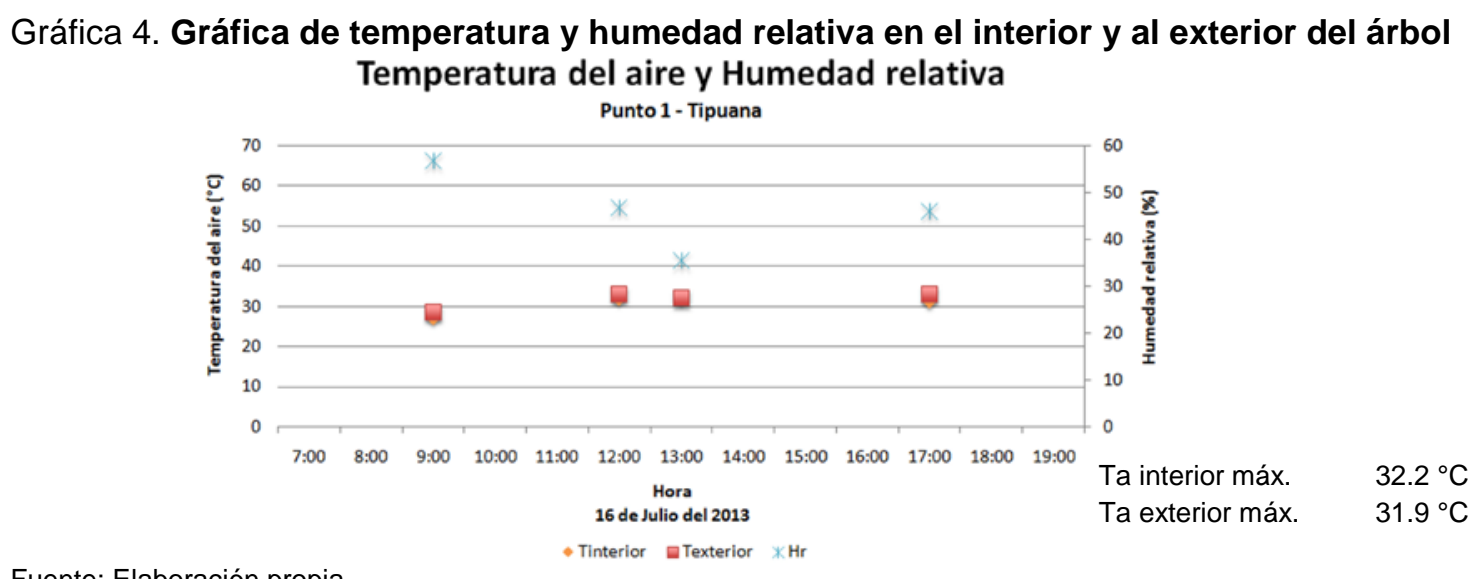

Fuente: Elaboración propia.

En la humedad relativa se observó al igual que el Almez que a primeras horas de la mañana una alta humedad, como explicamos anteriormente esta alta humedad es debido a la acumulación durante la madrugada que realiza la vegetación al ambiente. En el transcurso del día esta humedad disminuye por evaporación. A las 17:00h se registró una disminución de Ta y un aumento de humedad absoluta por evapotranspiración del árbol. En este caso La Tipuana creó menos aportaciones en relación al Almez. (Gráfica 4).

Tomando en cuenta lo explicado anteriormente sobre el estrés térmico. Evaluamos la Temperatura radiante, se registró una $\mathrm{Tr}$ exterior máxima de $59.4^{\circ} \mathrm{C}$ y una $\mathrm{Tr}$ exterior mínima de $27.9^{\circ} \mathrm{C}$. Sin embargo la $\mathrm{Tr}$ interior máxima fue de $37^{\circ} \mathrm{C}$ y una $\mathrm{Tr}$ interior mínima de $27.2^{\circ} \mathrm{C}$. Con una diferencia de $\operatorname{Tr}$ interior/exterior de $22.4{ }^{\circ} \mathrm{C}$ a las $13: 00 \mathrm{hrs}$ (Gráfica 5). Con una $\mathrm{Tr}$ exterior media de $48.6{ }^{\circ} \mathrm{C}$ y una $\mathrm{Tr}$ interior media de $33.5^{\circ} \mathrm{C}$. Y una diferencia promedio de la Tr interior/exterior de $15.2^{\circ} \mathrm{C}$.

\section{Gráfica 5. Gráfica de temperatura radiante en el interior y al exterior del árbol. Temperatura Radiante}

Punto 1- Tipuana

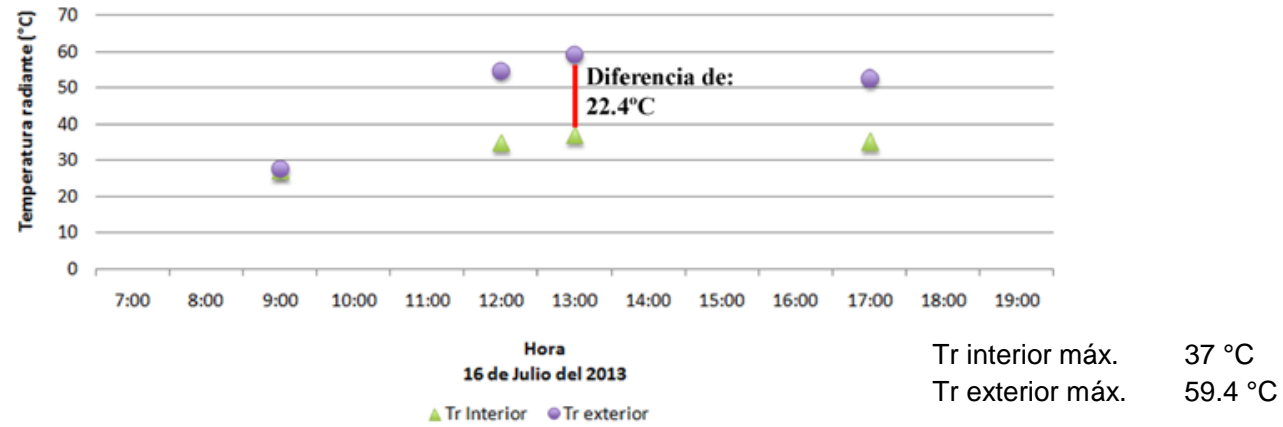

Fuente: Elaboración propia.

Podemos ver que a las $13.00 \mathrm{~h}$ existe una disminución de aportaciones térmicas $\left(22.4^{\circ} \mathrm{C}\right)$, permitiendo que a esta hora bajo la sombra del árbol, que el cuerpo realice su regulación de temperatura logrando estar en confort. 
En la iluminancia $(E)$ Se registró una $E$ exterior máxima de 110000 lx y una E exterior mínima de $5020 \mathrm{~lx}$, por lo que en este momento se estaba en dis-confort visual al exterior. La $\mathrm{E}$ interior máxima registrada fue de 3700 lx y la $E$ interior mínima fue de 1907 Ix, por lo que el usuario estaba en confort visual en relación al exterior. La $E$ interior/exterior se registró una diferencia de hasta $106300 \mathrm{Ix}$ a las 13:00 hrs. (gráfica 6). Con una E exterior media de $74055 \mathrm{~lx}$ y E interior media de $2401 \mathrm{~lx}$. Llegando a tener una diferencia de la $\mathrm{E}$ interior/exterior media de $716534 \mathrm{~lx}$.

\section{Gráfica 6. Gráfica de iluminancia en el interior y al exterior del árbol.}

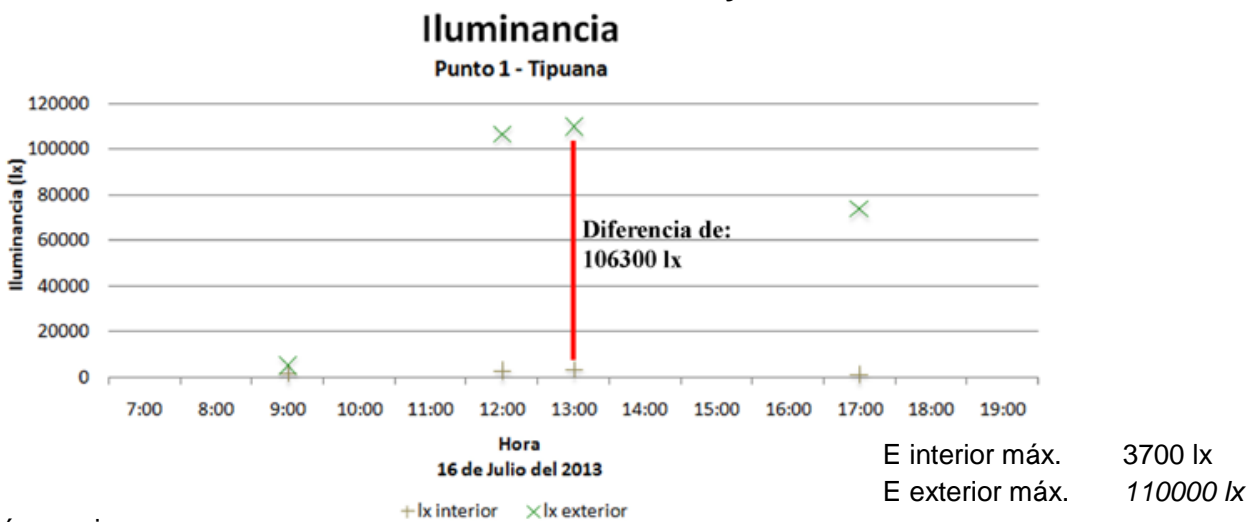

Fuente: Elaboración propia.

E exterior máx. 110000 lx

Estudio de HDR: En el caso de la Tipuana podemos observar a través del estudio una sombra homogénea, donde en las horas de la mañana y medio día no permite el paso de luz, pero sin embargo a horas de la tarde sí que permite el paso de la luz y esto se debe a su inclinación foliar (disposición de las hojas en la copa del árbol).

Si observamos las imágenes (Figura 6), dentro de la copa del árbol, se presentan valores de confort en relación a los valores exteriores. Por otra parte, los arbustos realizan la función de protección de zonas donde el árbol no podría proteger en horas donde el sol está más al horizonte. El comportamiento de la radiación solar en este caso sería igual que la iluminancia y luminancia.

Figura 6. Punto de medición 1. Tipuana Hora 9:00am, 13:00 y 17:00 hr.

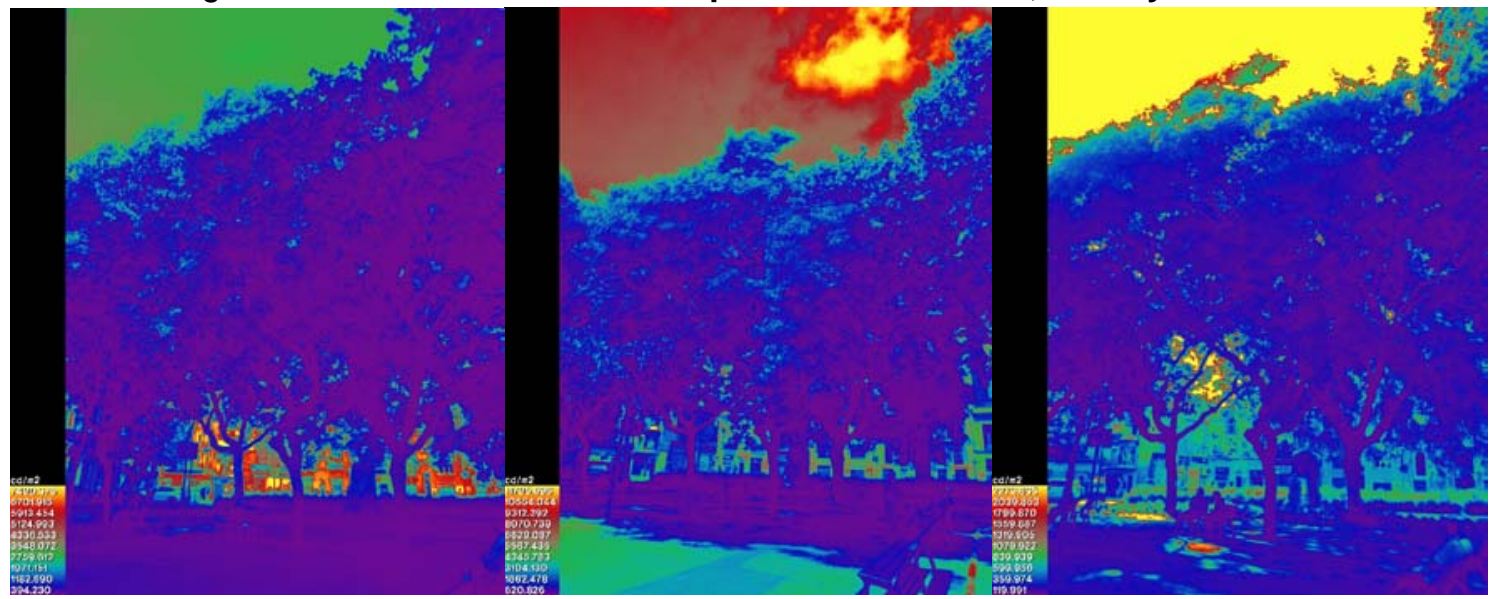

Fuente: Elaboración propia con base en Jaloxa. 
En el caso de la Tipuana podríamos asumir que durante las horas del día (9:00h -13:00h) debajo de la copa del árbol no penetrará la radiación debido a la densidad de las copa. Sin embargo, a las 17:00h penetra un porciento de radiación como bien explicamos anteriormente debido a la inclinación foliar.

\section{Conclusiones obtenidas}

Para el análisis de los demás puntos de mediciones se realizó el mismo procedimiento de las mediciones de los parámetros de confort. Como pudimos observar en el desarrollo detallado de las medidas del punto 1 , la $\mathrm{Tr}$ y la $\mathrm{E}$ son los datos más importantes a considerar. Por este motivo, se ha resumido en las tablas 4 hasta la 8 los datos obtenidos de la $\operatorname{Tr}$ interior (Tri), $\mathrm{Tr}$ exterior (Tre), $\mathrm{E}$ interior (Ei), E exterior (Ee) y la diferencia interior/exterior ( $\Delta \operatorname{Tr}$ y $\Delta \mathrm{E})$.

\section{Punto 1}

Analizando las especies arbóreas de este punto (Figura 2) podemos describir que la Tipuana otorga más protección a la incidencia solar a las 13:00h, debido al tamaño de sus hojas (Hojas grandes y compuestas) y por la densidad de su follaje en la parte superior (sombra media), pero debido a la inclinación foliar esta protección se ve afectada a las 17:00hr, permitiendo el paso de la radiación solar creando aportaciones al ambiente.

Sin embargo, el Almez (Hojas tamaño medio y alternas Oval) con una abundante densidad de hojas (sombra densa) ofrece mayor protección a toda hora por la inclinación de sus hojas, las cuales están en posición más horizontal. Podemos concluir que el Almez es más factible para el uso urbano en zonas tales como parques, calles, paseos y ramblas. Se recomienda debido a la protección constante de la morfología urbana y el confort visual que ofrece.

Para el análisis del pavimento de hormigón, durante todo el día se registraron valores muy elevados de $\operatorname{Tr}$ y $E$, este tipo de pavimento tiene un alto nivel de aportaciones térmicas, logrando que el usuario no pueda regular su propia temperatura logrando el dis-confort.

\section{Punto 2}

En este punto de análisis el interés principal nació por las dos (2) especies arbóreas con características de morfología urbanas similares. Pero sin embargo, los usuarios optan estar más a la sombra del árbol de los Faroles. Se evaluó el porqué la distinción de uso entre ambas. (Figura 7) (Tabla 4). 
Figura 7. Passeig Sant Joan, Punto de medición 2, A. Amor y A. Faroles
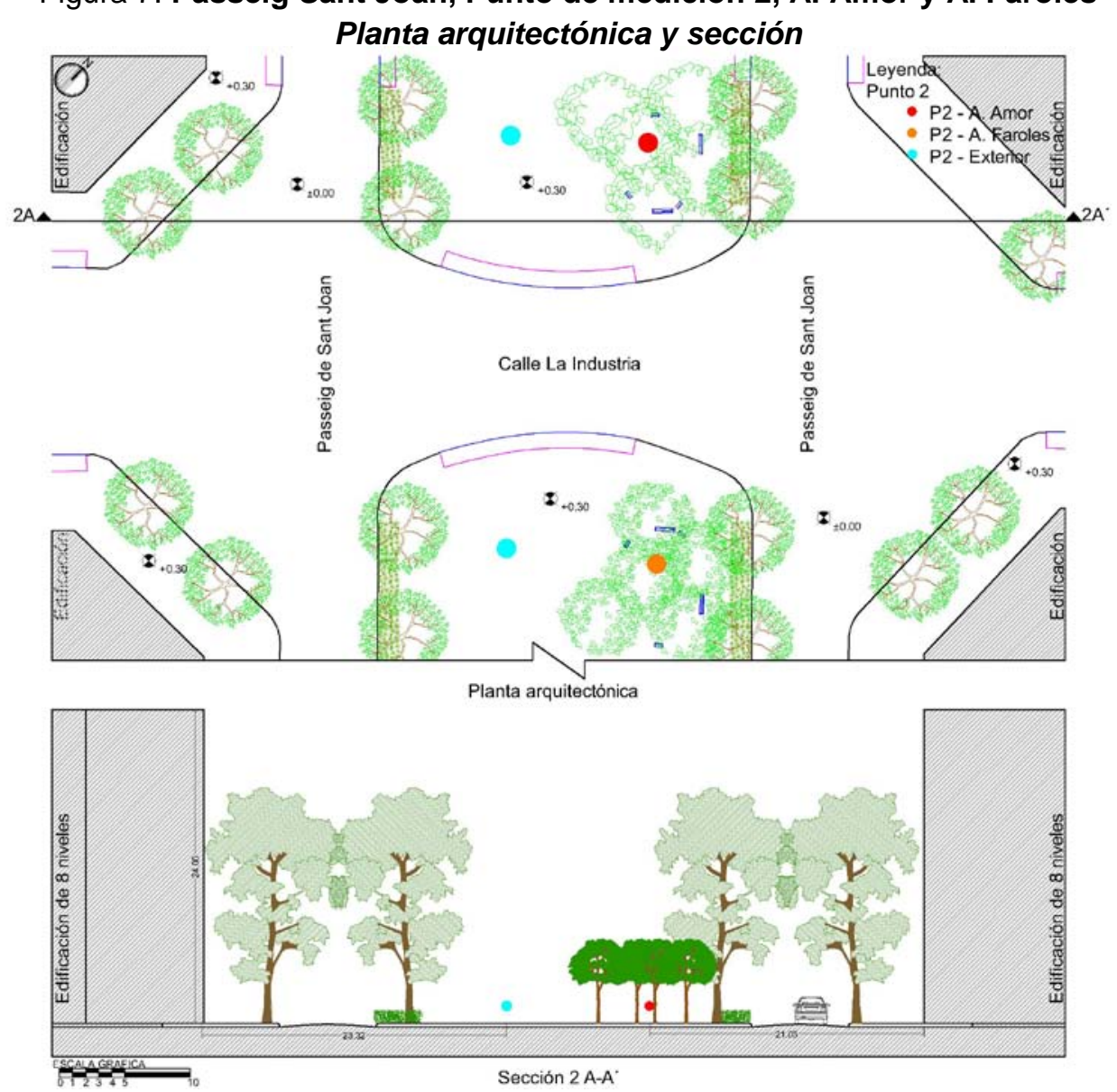

Fuente: Elaboración propia con base en Ajuntament de Barcelona.

Tabla 4. Datos de mediciones Punto 2. Á. del Amor y A. de los Faroles

\begin{tabular}{|c|c|c|c|c|c|c|}
\hline \multicolumn{7}{|c|}{ Punto 2} \\
\hline \multicolumn{7}{|c|}{ Árbol del amor } \\
\hline Hora & Tr Interior & Tr exterior & $\Delta \operatorname{Tr}$ & E interior & E exterior & $\Delta \mathrm{E}$ \\
\hline 09:00 & $27.3^{\circ} \mathrm{C}$ & $28.5^{\circ} \mathrm{C}$ & $1.2^{\circ} \mathrm{C}$ & $926 \mathrm{Ix}$ & $8010 \mathrm{~lx}$ & $3113 \mathrm{Ix}$ \\
\hline 13:00 & $36.5^{\circ} \mathrm{C}$ & $60.7^{\circ} \mathrm{C}$ & $24.2^{\circ} \mathrm{C}$ & $5190 \mathrm{Ix}$ & $111000 \mathrm{~lx}$ & $105810 \mathrm{~lx}$ \\
\hline $17: 00$ & $34.1^{\circ} \mathrm{C}$ & $57.0^{\circ} \mathrm{C}$ & $22.9^{\circ} \mathrm{C}$ & $2540 \mathrm{~lx}$ & $74000 \mathrm{Ix}$ & $71460 \mathrm{Ix}$ \\
\hline \multicolumn{7}{|c|}{ Árbol de los faroles } \\
\hline Hora & Tr Interior & Tr exterior & $\Delta \operatorname{Tr}$ & E interior & E exterior & $\Delta \mathrm{E}$ \\
\hline 09:00 & $26.0^{\circ} \mathrm{C}$ & $27.3^{\circ} \mathrm{C}$ & $1.3^{\circ} \mathrm{C}$ & $715 \mathrm{Ix}$ & $6850 \mathrm{~lx}$ & $6135 \mathrm{~lx}$ \\
\hline 13:00 & $32.0^{\circ} \mathrm{C}$ & $60.7^{\circ} \mathrm{C}$ & $28.7^{\circ} \mathrm{C}$ & $1330 \mathrm{~lx}$ & $111000 \mathrm{~lx}$ & $109670 \mathrm{Ix}$ \\
\hline $17: 00$ & $33.1^{\circ} \mathrm{C}$ & $57.0^{\circ} \mathrm{C}$ & $23.9^{\circ} \mathrm{C}$ & $1900 \mathrm{Ix}$ & $74000 \mathrm{Ix}$ & $72100 \mathrm{~lx}$ \\
\hline
\end{tabular}

Fuente: Elaboración propia.

El resultado obtenido es que el árbol de los Faroles es el que ofrece mayor protección por el tamaño de sus hojas (hojas grandes) podemos observarlo en la $\operatorname{Tr}$ y E interior/exterior donde se registraron altos valores de diferencia. Aunque ambas especies son de sombra media y su 
ramaje son diferentes tortuoso (A. de los Faroles) y expandido (A. del Amor) el tamaño de las hojas compensa la funcionabilidad que debería cumplir el ramaje.

Concluyendo que la preferencia de los usuarios por el $A$. de los Faroles es debido a que logra disminuir más las aportaciones térmicas y visuales, logrando de esta forma que bajo su sombra se pueda disfrutar del espacio exterior sin percibir fuertemente las incidencias ambientales. Siendo el A. de los Faroles el más recomendable para zonas como plazas, jardines y paseo.

\section{Punto 3}

El criterio de selección de este punto es debido a que, bajo la copa de estas tres especies existen zonas de estancia creando el interés de conocer cual especie ofrece mayor confort. Analizando las especies podemos concluir que el Almez y el Plátano ofrecen mayor protección debido al tamaño de sus hojas (hojas media y grande), inclinación foliar (hojas más horizontales), densidad y diámetro de su follaje (sombra densa). Convirtiéndolas en especies idóneas para uso en la morfología urbana en zonas como parques, calles amplias, ramblas o paseo. (Figura 8) (Tabla 5).

Figura 8. Passeig Sant Joan, Punto de medición 3. Almez, Plátano y Tilia Planta arquitectónica y sección
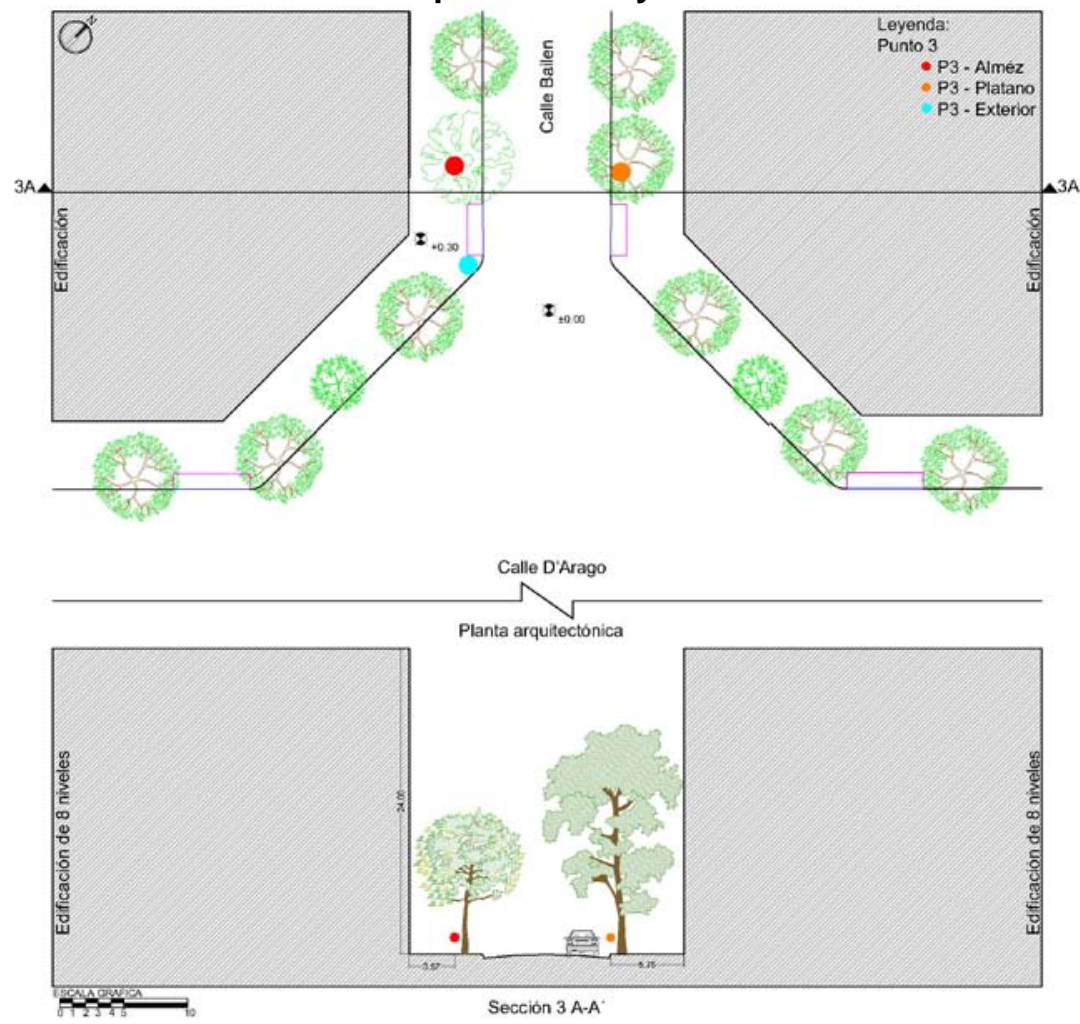

Fuente: Elaboración propia con base en Ajuntament de Barcelona. 
Tabla 5. Datos de mediciones Punto 3. Almez, Plátano y Tilia

\begin{tabular}{|c|c|c|c|c|c|c|}
\hline \multicolumn{7}{|c|}{ Punto 3} \\
\hline \multicolumn{7}{|c|}{ Almez } \\
\hline Hora & Tr Interior & Tr exterior & $\Delta \mathrm{Tr}$ & E interior & E exterior & $\Delta \mathrm{E}$ \\
\hline 09:00 & $30.8^{\circ} \mathrm{C}$ & $32.5^{\circ} \mathrm{C}$ & $1.7^{\circ} \mathrm{C}$ & 1484 Ix & $47000 \mathrm{~lx}$ & $45516 \mathrm{Ix}$ \\
\hline $14: 00$ & $32.5^{\circ} \mathrm{C}$ & $53.0^{\circ} \mathrm{C}$ & $20.5^{\circ} \mathrm{C}$ & $2480 \mathrm{Ix}$ & $108000 \mathrm{~lx}$ & $105520 \mathrm{Ix}$ \\
\hline $17: 00$ & $30.6^{\circ} \mathrm{C}$ & $52.1^{\circ} \mathrm{C}$ & $21.5^{\circ} \mathrm{C}$ & $1100 \mathrm{Ix}$ & $72000 \mathrm{~lx}$ & $70900 \mathrm{Ix}$ \\
\hline \multicolumn{7}{|c|}{ Plátano } \\
\hline Hora & Tr Interior & Tr exterior & $\Delta \operatorname{Tr}$ & E interior & E exterior & $\Delta \mathrm{E}$ \\
\hline 09:00 & $29.4^{\circ} \mathrm{C}$ & $34.5^{\circ} \mathrm{C}$ & $5.1^{\circ} \mathrm{C}$ & $3130 \mathrm{~lx}$ & $47000 \mathrm{~lx}$ & $43870 \mathrm{Ix}$ \\
\hline $14: 00$ & $32.2^{\circ} \mathrm{C}$ & $53.0^{\circ} \mathrm{C}$ & $20.8^{\circ} \mathrm{C}$ & $3500 \mathrm{Ix}$ & $108000 \mathrm{~lx}$ & $104500 \mathrm{Ix}$ \\
\hline $17: 00$ & $33.8^{\circ} \mathrm{C}$ & $52.1^{\circ} \mathrm{C}$ & $18.3^{\circ} \mathrm{C}$ & $2960 \mathrm{Ix}$ & $72000 \mathrm{Ix}$ & $69040 \mathrm{~lx}$ \\
\hline \multicolumn{7}{|c|}{ Tilia } \\
\hline Hora & Tr Interior & Tr exterior & $\Delta T r$ & E interior & E exterior & $\Delta \mathrm{E}$ \\
\hline 09:00 & $27.3^{\circ} \mathrm{C}$ & $30.8^{\circ} \mathrm{C}$ & $3.5^{\circ} \mathrm{C}$ & $3130 \mathrm{Ix}$ & $47000 \mathrm{~lx}$ & $43870 \mathrm{~lx}$ \\
\hline $14: 00$ & $38.0^{\circ} \mathrm{C}$ & $56.4^{\circ} \mathrm{C}$ & $18.4^{\circ} \mathrm{C}$ & $3500 \mathrm{Ix}$ & $108000 \mathrm{~lx}$ & $104000 \mathrm{Ix}$ \\
\hline $17: 00$ & $37.7^{\circ} \mathrm{C}$ & $46.3^{\circ} \mathrm{C}$ & $8.6^{\circ} \mathrm{C}$ & $2960 \mathrm{Ix}$ & $72000 \mathrm{~lx}$ & $56500 \mathrm{~lx}$ \\
\hline
\end{tabular}

Fuente: Elaboración propia.

Evaluando el uso actual de estas dos especies arbóreas podemos concluir que la utilización de ambas como elementos de protección de morfología urbana. Por ejemplo, la calle Bailen se realiza aplicaciones de dos especies arbóreas diferentes que logran en conjunto un resultado final satisfactorio. El Almez con una altura que comprende de 10 a $20 \mathrm{mt}$, y un diámetro de follaje logra proteger durante todo el año las superficies urbanas. Sin embargo, el Plátano con una altura de 20 a $30 \mathrm{mt}$, cumple la función de proteger la calle desde un punto más alto. Ambas especies en conjunto logran en estaciones donde la incidencia solar es mayor que las calles estén totalmente impermeables.

Por otra parte, en épocas donde la incidencia solar es menor y es requerida por el usuario el Plátano pasa a ser permeable y el Almez conserva sus hojas pero con menos densidad. Logrando como resultado final que gracias a sus características naturales estas dos especies logren proteger y crear confort sin necesidad de elementos más allá que la naturaleza. Por otra parte, la Tilia ofreció menos protección solar debido a su densidad y diámetro de follaje, por esto se recomienda para uso urbano de soporte de otra especie arbórea o de forma ornamental.

\section{Punto 4}

La Plaza Tetuán fue seleccionada por su tipo de pavimento que es el cauce, la variedad ${ }^{17}$ de tres tipos especies arbóreas en el punto de estudio y la versatilidad de edades de los usuarios que recurren a esta plaza. (Figura 9) (Tabla 6).

\footnotetext{
${ }^{17}$ En este punto están las dos especies estudiadas en el punto I, pero con un tipo de pavimento distinto.
} 
Figura 9. Passeig Sant Joan, Punto de medición 4. Tipuana, Palmera canaria y Almez Planta arquitectónica y sección
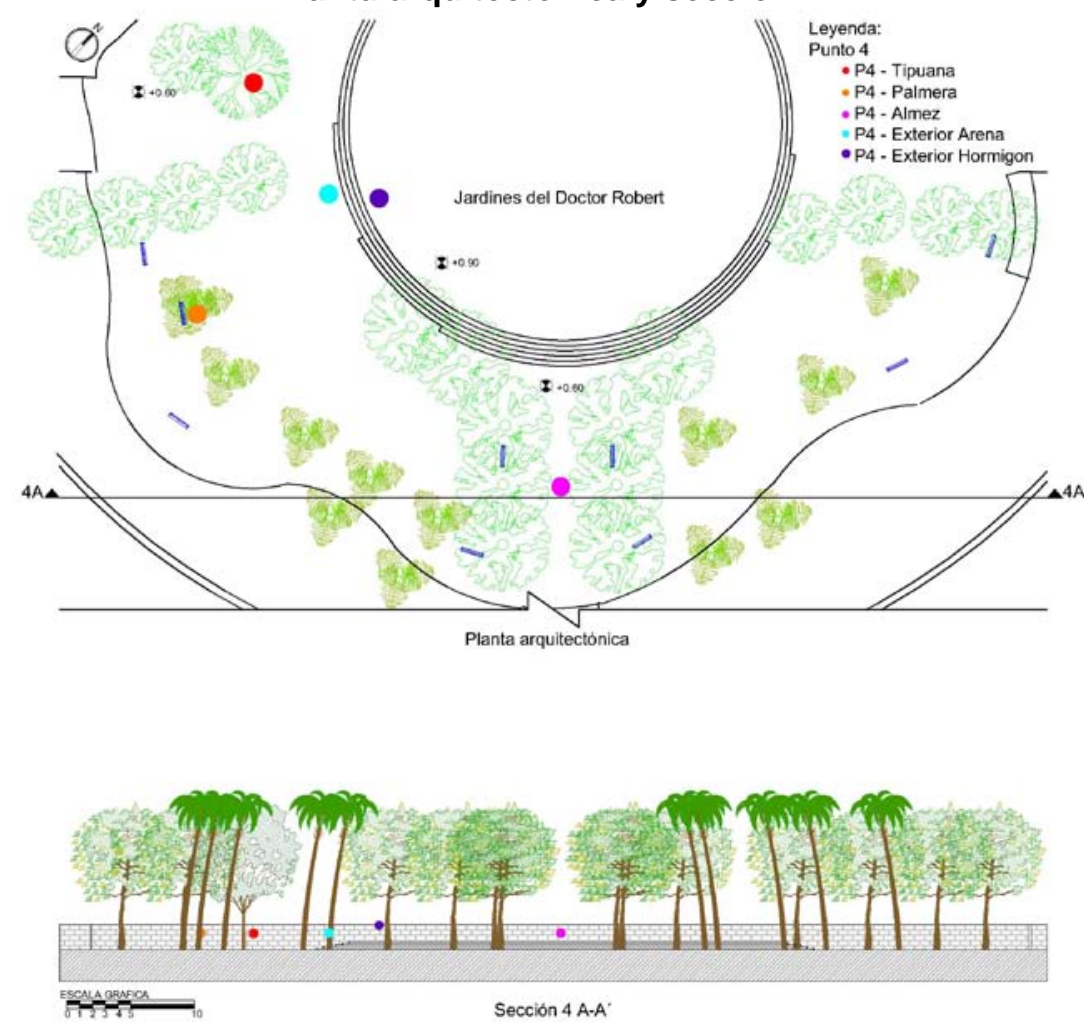

Fuente: Elaboración propia con base en Ajuntament de Barcelona.

Tabla 6. Datos de mediciones Punto 4. Tipuana, Palmera canaria y Almez

\begin{tabular}{|c|c|c|c|c|c|c|}
\hline \multicolumn{7}{|c|}{ Punto 4} \\
\hline \multicolumn{7}{|c|}{ Tipuana } \\
\hline Hora & Tr Interior & Tr exterior & $\Delta T r$ & E interior & E exterior & $\Delta \mathrm{E}$ \\
\hline 09:00 & $27.4^{\circ} \mathrm{C}$ & $36.6^{\circ} \mathrm{C}$ & $9.2^{\circ} \mathrm{C}$ & $3600 \mathrm{Ix}$ & $5500 \mathrm{Ix}$ & $1900 \mathrm{Ix}$ \\
\hline $14: 00$ & $32.0^{\circ} \mathrm{C}$ & $55.0^{\circ} \mathrm{C}$ & $23.0^{\circ} \mathrm{C}$ & $5500 \mathrm{~lx}$ & $130000 \mathrm{~lx}$ & $124500 \mathrm{~lx}$ \\
\hline $17: 00$ & $32.5^{\circ} \mathrm{C}$ & $54.0^{\circ} \mathrm{C}$ & $21.5^{\circ} \mathrm{C}$ & $4000 \mathrm{Ix}$ & $108000 \mathrm{~lx}$ & $104000 \mathrm{Ix}$ \\
\hline \multicolumn{7}{|c|}{ Palmera canaria } \\
\hline Hora & Tr Interior & Tr exterior & $\Delta T r$ & E interior & E exterior & $\Delta \mathrm{E}$ \\
\hline 09:00 & $24.3^{\circ} \mathrm{C}$ & $36.6^{\circ} \mathrm{C}$ & $12.3^{\circ} \mathrm{C}$ & $6540 \mathrm{~lx}$ & $5500 \mathrm{~lx}$ & $1040 \mathrm{Ix}$ \\
\hline $14: 00$ & $37.2^{\circ} \mathrm{C}$ & $55.0^{\circ} \mathrm{C}$ & $17.8^{\circ} \mathrm{C}$ & $16100 \mathrm{~lx}$ & $130000 \mathrm{~lx}$ & $113900 \mathrm{~lx}$ \\
\hline $17: 00$ & $34.0^{\circ} \mathrm{C}$ & $54.0^{\circ} \mathrm{C}$ & $20.0^{\circ} \mathrm{C}$ & $10000 \mathrm{Ix}$ & $108000 \mathrm{~lx}$ & $98000 \mathrm{~lx}$ \\
\hline \multicolumn{7}{|c|}{ Almez } \\
\hline Hora & Tr Interior & Tr exterior & $\Delta T r$ & E interior & E exterior & $\Delta \mathrm{E}$ \\
\hline $09: 00$ & $23.8^{\circ} \mathrm{C}$ & $36.6^{\circ} \mathrm{C}$ & $12.8^{\circ} \mathrm{C}$ & $2450 \mathrm{Ix}$ & $5500 \mathrm{Ix}$ & $43870 \mathrm{Ix}$ \\
\hline $14: 00$ & $31.2^{\circ} \mathrm{C}$ & $55.0^{\circ} \mathrm{C}$ & $23.8^{\circ} \mathrm{C}$ & $9200 \mathrm{Ix}$ & $130000 \mathrm{~lx}$ & $120800 \mathrm{~lx}$ \\
\hline $17: 00$ & $33.0^{\circ} \mathrm{C}$ & $54.0^{\circ} \mathrm{C}$ & $21.0^{\circ} \mathrm{C}$ & $2900 \mathrm{Ix}$ & $108000 \mathrm{~lx}$ & $105100 \mathrm{~lx}$ \\
\hline
\end{tabular}

Fuente: Elaboración propia. 
Analizando las especies arbóreas, el Almez y la Tipuana por sus características como el tamaño de sus hojas (hojas media y grande), densidad y diámetro de su follaje (sombra densa y media) ofrecen un alto nivel de protección, por lo que son recomendadas para zonas como parques, calles amplias, ramblas y paseo. Analizando la distribución actual del espacio podemos decir que el Almez es más propicio para zonas de estancias más prolongadas y la Tipuana mas para zonas de tránsito peatonal.

Sin embargo, la Palmera canaria ofrece bajos niveles de protección, debido a su diámetro de follaje, por lo que se recomienda para uso urbano costero. En el caso de uso urbano actual en la Plaza Tetuán seria más factible sustituir las palmeras por el Almez.

Para el análisis de los tipos de pavimento el cauce, registró $\operatorname{Tr}$ y E muy elevadas por lo que sus aportaciones térmicas tiene niveles muy alto creando el dis-confort.

\section{Punto 5}

La Plaza Tetuán fue seleccionada por su tipo de pavimento que es el cauce, la variedad ${ }^{18}$ de tres tipos especies arbóreas en el punto de estudio y la versatilidad de edades de los usuarios que recurren a esta plaza. (Figura 9) (Tabla 6).

${ }^{18}$ En este punto están las dos especies estudiadas en el punto I, pero con un tipo de pavimento distinto. 
Figura 10. Passeig Sant Joan, Punto de medición Plátano

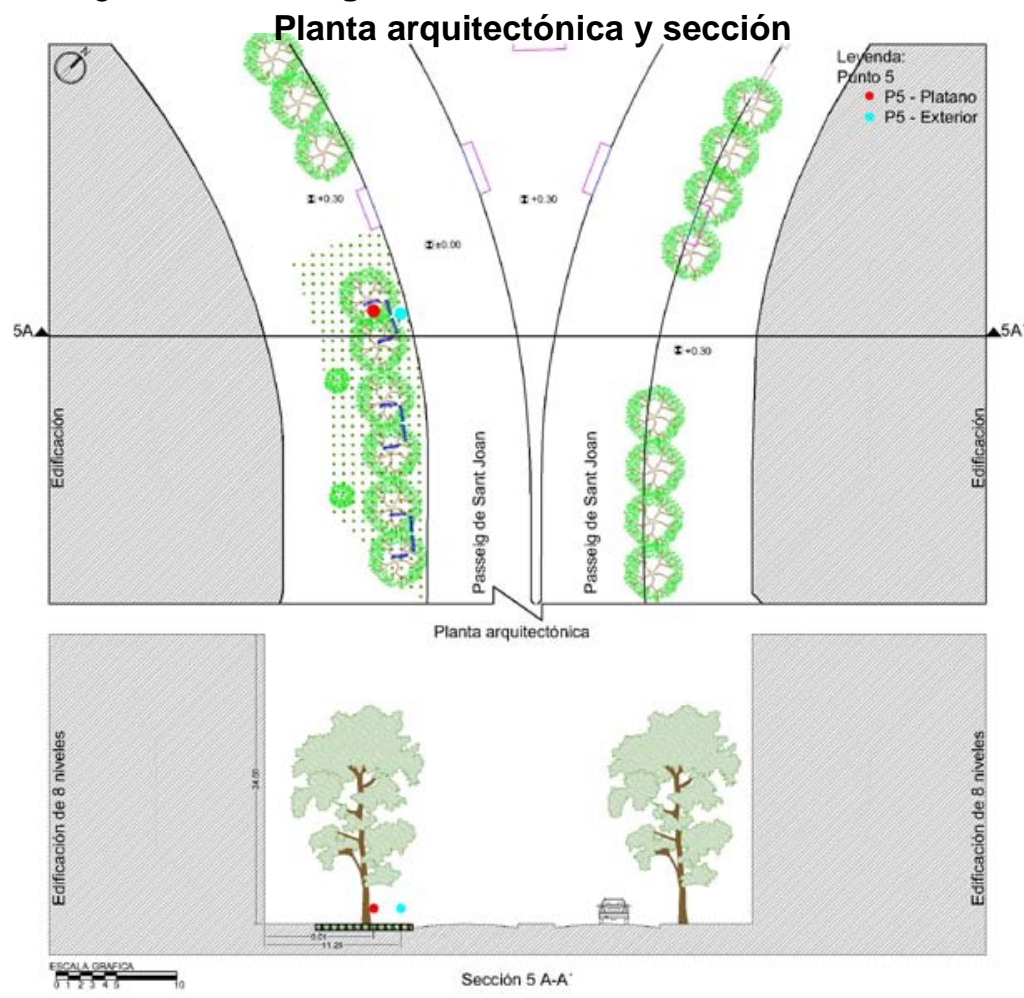

Fuente: Elaboración propia con base en Ajuntament de Barcelona.

Tabla 7. Datos de mediciones Punto 5. Plátano y A. Varios (Arbustivos y Plátano)

\begin{tabular}{|c|c|c|c|c|c|c|}
\hline \multicolumn{7}{|c|}{ Plátano } \\
\hline Hora & Tr Interior & Tr exterior & $\Delta \mathrm{Tr}$ & E interior & E exterior & $\Delta \mathrm{E}$ \\
\hline 09:00 & $26.3^{\circ} \mathrm{C}$ & $30.2^{\circ} \mathrm{C}$ & $3.9^{\circ} \mathrm{C}$ & $6400 \mathrm{Ix}$ & $35000 \mathrm{~lx}$ & $28600 \mathrm{~lx}$ \\
\hline $14: 00$ & $30.8^{\circ} \mathrm{C}$ & $50.3^{\circ} \mathrm{C}$ & $19.5^{\circ} \mathrm{C}$ & $4300 \mathrm{Ix}$ & $120000 \mathrm{Ix}$ & $115700 \mathrm{~lx}$ \\
\hline $17: 00$ & $29.5^{\circ} \mathrm{C}$ & $40.5^{\circ} \mathrm{C}$ & $11.0^{\circ} \mathrm{C}$ & $2700 \mathrm{Ix}$ & $74000 \mathrm{~lx}$ & $71300 \mathrm{Ix}$ \\
\hline \multicolumn{7}{|c|}{ Arboles Varios (arbustivo y plátano) } \\
\hline Hora & Tr Interior & Tr exterior & $\Delta \operatorname{Tr}$ & E interior & E exterior & $\Delta \mathrm{E}$ \\
\hline 09:00 & $24.0^{\circ} \mathrm{C}$ & $30.2^{\circ} \mathrm{C}$ & $6.2^{\circ} \mathrm{C}$ & $2700 \mathrm{Ix}$ & $35000 \mathrm{~lx}$ & $32300 \mathrm{~lx}$ \\
\hline $14: 00$ & $27.1^{\circ} \mathrm{C}$ & $50.3^{\circ} \mathrm{C}$ & $23.2^{\circ} \mathrm{C}$ & $12600 \mathrm{Ix}$ & $120000 \mathrm{~lx}$ & $107400 \mathrm{~lx}$ \\
\hline $17: 00$ & $31.2^{\circ} \mathrm{C}$ & $40.5^{\circ} \mathrm{C}$ & $9.3^{\circ} \mathrm{C}$ & $6200 \mathrm{Ix}$ & $74000 \mathrm{~lx}$ & $67800 \mathrm{~lx}$ \\
\hline
\end{tabular}

Fuente: Elaboración propia.

Esta morfología urbana fue la rediseñada por la Arq. Domenech (Domenech 2008). El criterio de selección ha sido el análisis de cómo el confort es afectado en la morfología urbana al implementar o no arbustivos, la variedad de tipo de pavimento (el hormigón y césped) con las mismas especies arbóreas y por ser zonas de uso constante por los usuarios. (Figura 10) (Tabla 7). 
Como resultado se puede observar en la tabla 8 que los arbustivos logran proteger la zona inferior del árbol en el ocaso, logrando mayor protección. La diferencia de Tr obtenida de las mediciones realizadas en el interior y exterior de la copa del árbol con arbustos es de hasta $23.2^{\circ} \mathrm{C}$ a las 14:00h (Tabla 8). En la E se registraron niveles de confort visual. El tipo de pavimento evaluado es el hormigón con césped, los valores de $\operatorname{Tr}$ arrojo resultados muy inferior en relación al hormigón y el cauce, siendo este el más factible para la zona urbana. Evaluando el actual diseño de esta zona de Passeig Sant Joan, se considera que es un modelo de morfología urbana.

\section{Conclusiones Generales}

La motivación principal para la realización de esta investigación, es conocer como la vegetación influye en el confort micro climático en la morfología urbana. Tomando como base los resultados analizados, nos cuestionamos ¿Es la vegetación la mejor herramienta para la mitigación de las aportaciones térmica en la morfología urbana? La respuesta es sí, la vegetación es la herramienta más idónea para poder mitigar la situación de dis-confort a nivel urbano, podemos observar en los resultados obtenidos como disminuye las aportaciones debido a la calidad de la sombra del árbol. Durante la metodología de campo se observó diferentes tipos de especies arbóreas en la misma morfología urbana, donde el usuario optaba por aquella que ofrecía más confort ante las incidencias ambientales. Siendo el usuario el que ofrece la respuesta de cual especie es más adecuada.

Dando como resultante la segunda interrogante ¿Las características de la vegetación influyen en el confort y en que estas aportaciones térmicas sean más o menos significativas? Luego de analizados los resultados podemos concluir que las características sí influyen, debido a que cada especie según su zona bioclimática puede variar su respuesta ante el ambiente como la calidad de protección que pueda ofrecer. Tenemos como ejemplo de los casos de estudio, la calle Bailen, donde las especies analizadas fueron el Almez, el Plátano y la Tilia. En este estudio podemos ver como el Plátano tiene una respuesta completamente distinta ante los cambios climáticos en comparación con el Almez y la Tilia. Siendo esto favorable ya que entre ambas compensan el confort ante las incidencias climáticas de cada estación del año.

Analizado los casos surgieron las siguientes interrogantes ¿Cuál de las especies de este estudio son las más recomendadas para el uso urbano? Podemos recomendar para uso en la morfología urbana, tomando como base el análisis realizado las especies como el Almez por su nivel de permeabilidad, el Árbol de los Faroles por su diámetro de protección y el tipo de hoja, el Plátano por su altura, sombra y versatilidad ante las estaciones climáticas, la Tipuana para uso de zonas de transito y como ultimo la implementación de arbustivos con árboles en zonas de recreación y larga estancia. ¿De los pavimentos evaluados cual es más factible para el diseño urbano? Se estudiaron tres tipos el hormigón, el cauce y el hormigón con césped. De estos tres tipos el más recomendado es el hormigón con césped, debido a los niveles de confort que ofrece por la disminución de aportaciones térmicas de temperatura radiante y de lluminancia. Esto sucede debió a que el césped recibe la radiación convirtiéndola en fotosíntesis y absorbiendo la otra parte de la energía. 
Como toda investigación que busca la respuesta a situaciones, de igual forma crea propuestas de investigaciones futuras, ¿Qué futuras investigaciones podrían realizarse sobre el tema expuesto? Líneas de investigación de "cómo la morfología del arbolado urbano es un elemento de vital importancia que debe ser considerado en el diseño", "Como cada clima implementa especies arbóreas a nivel urbano de otras zonas climáticas y como esto podría afectar a nivel climático o monetario" entre otras que vayan enfocada a como cada especie es un organismo propio que ofrece respuesta a su entorno inmediato.

Podemos concluir que la vegetación tiene características como el tipo de hoja, la fotosíntesis, el ramaje, la forma de follaje, densidad de follaje, la inclinación foliar y evapotranspiración que son las cuales definen que cantidad de protección y qué cantidad de humedad recibe el ambiente. Es una excelente herramienta para crea microclimas exteriores, la clave principal es conocer las características que ofrecen el arbolado y las necesidades principales en el diseño. Es la principal herramienta para mitigar la isla de calor, gracias a que disminuye las aportaciones en la morfología urbana. Y se recomienda como tipo de pavimento para uso en la morfología urbana el pavimento de hormigón con césped, ya que la combinación de ambos permite tanto que el césped cumpla su función de absorción de la radiación y del hormigón como elemento de resistencia.

\section{Agradecimientos}

A Dios ante todo, mis familiares, mis amigos, al Ministerio de Educación de la República Dominicana, (MESCYT), a la Dirección de Gestión Ambiental y la Ing. Anyelina Aquino, A Srta. Marianna Szabo, a la Dr. Helena Coch y mi grupo de profesores del AEM 2012-2013 y al grupo AEM 2012-2013.

\section{Bibliografía}

ABREU, L. V.; LABAKI, L.L. y MATZARAKIS, A. Bioclimate provide by shade trees as factor in urban and architectural planning in tropical climates - the case of Campinas, Brazil. En: Smart and sustainable built environments: 1-7, 2012.

ARBOIT, M.E. Permeabilidad del arbolado urbano a la radiación solłfrstudio de dos especies representativas en entornos urbanos de baja densidad del Área Metropolitana de Mendoza, Argentina. En: Hábitat Sustentable, 3: 3-18 [en línea] Disponible en: <http://www.revistahabitatsustentable.cl/ojs/index.php/arquitec/article/view/591/369>. 2013.

ARMSON, D.; STRINGER, P. y ENNOS, A.R. The effect of tree shade and grass on surface and globe temperatures in an urban area. En: Urban Forestry \& Urban Greening: 11 (3): 245255 [en línea] Fecha de consulta: 8 de marzo de 2013. ISSN 16188667. DOI 10.1016/j.ufug.2012.05.002. Disponible <http://linkinghub.elsevier.com/retrieve/pii/S1618866712000611>. 2012 
BARBIRATO, G.M.; CAMAÚBA, S. y ALMEIDA, T. Microclimatic conditions of urban public spaces in a tropical city [en línea] Disponible en: <http://nargeo.geo.uni.lodz.pl/-icuc5/text/O_2_5.pdf>. 1988.

CAMINERO, H.H. Incidencia termica y luminca de los pavimentos exteriores en el interior de la vivienda, para clima calido - humedo caso Santo DOmingo, Republica Dominicana. Barcelona, Universidad Politécnica de Cataluña, 2012.

CANTÓN, M.; CORTEGOSO, J.L. y DE ROSA, C. Evaluación energética - ambiental del bosque urbano: desarrollo y puesta a punto de un método de análisis [en línea]. Disponible en: <http://www.cricyt.edu.ar/asades/modulos/averma/trabajos/2000/2000-t001-a003.pdf>. 2000.

CATALUNYA, G. Atlas de radiació solar a Catalunya [en línea] Disponible en: $<$ http://www20.gencat.cat/docs/icaen/02_EnergiesRenovables/Documents/Arxius/Atlasde radiaciosolar.pdf>. 2001.

CATALUNYA, G. Meteocat [en línea]. Fecha de consulta: 28 de juliode 2013. Disponible en: <http://www.meteo.cat/xema/AppJava/Mapper.do>. 2013.

DE BARCELONA, A. Gestión del arbolado viario de Barcelona [en línea]. Disponible en: $<$ https://w110.bcn.cat/MediAmbient/Continguts/Vectors_Ambientals/Espais_Verds/Documents/ Traduccions/Plangestionarboladoviariobcn_cast.pdf>. 2011.

DOMENECH, L. Lola Domenech [en línea] Fecha de consulta: 4 de junio de 2013. Disponible en: <http://www.loladomenech.com/es/estudio.php>. 2008.

ECHAVE, C. Ver des de la ciudad. Potencial de habitabilidad térmica en espacios urbano. [en línea] Disponible en: <http://issuu.com/cynthiaechave/docs/verdes_de_la_ciudad_cynthia_echave>. 2007.

JALOXA. Jaloxa [en línea] Fecha de consulta: 2 de agosto de 2013. Disponible en: <http://www.jaloxa.eu/webhdr/roll-your-own.shtml>. 2012.

MAKAREMI, N.; SALLEH, E.; JAAFAR, M.Z. y GHAFFARIANHOSEINI, A. Thermal comfort conditions of shaded outdoor spaces in hot and humid climate of Malaysia.En: Building and Environment, 48: 7-14 [en línea] Fecha de consulta: 2 de mayo de 2013. ISSN 03601323. DOI 10.1016/j.buildenv.2011.07.024. Disponible en: <http://linkinghub.elsevier.com/retrieve/pii/S0360132311002381>. 2012.

MELO, G.; CARNAÚBA, S. y DE ALMEIDA, T. Microclimatic conditions of urban public in a tropical city [en línea] Disponible en: <http://nargeo.geo.uni.lodz.pl/ icuc5/text/O_2_5.pdf>. 1988.

NAVÉS, F. Arquitectura del paisaje natural. Barcelona, Omega, 2005.

OCHOA, J.M. La Vegetacion como instrumento para el control microclimatico. Barcelona, Universidad Politecnica de Catalunya, 1999. 
ROSAS, M.A. El confort ambiental de las circulaciones peatonales en el litoral costero de Barcelona, España. Barcelona, Universitat Politècnica de Catalunya, 2010.

SERRA, R. y COCH, H. Arquitectura y energía natural. Barcelona, 1995.

TUMINI, I. El microclima urbano en los espacios abiertos. Estudio de casos en Madrid [en línea] Disponible en: <http://oa.upm.es/14893/1/IRINA_TUMINI.pdf>. 2012.

VARGAS, K.E.; MCPHERSON, E.G.; SIMPSON, J.R.; PEPER, P.J.; GARDNER, S.L. y XIAO, Q. City of Honolulu, Hawai ' $i$ Municipal Forest Resource Analysis [en línea] Fecha de consulta: 20 de mayo de 2013.2 Disponible en: <http://www.fs.fed.us/psw/programs/uesd/uep/products/2/psw_cufr724_HNL_MFRA.pdf>. 2007. 